\title{
Supporting Information: Coherent-to-Incoherent Transition of Molecular Fluorescence Controlled by Surface Plasmon Polaritons
}

\author{
Siwei Wang, ${ }^{\dagger, \ddagger}$ Gregory D. Scholes, ${ }^{*, \neq}$ and Liang-Yan Hsu ${ }^{*, \dagger}$ \\ $\dagger$ Institute of Atomic and Molecular Sciences, Academia Sinica, Taipei 10617, Taiwan \\ $\ddagger$ Department of Chemistry, Princeton University, Princeton, New Jersey 08544, United \\ States
}

E-mail: gscholes@princeton.edu; lyhsu@gate.sinica.edu.tw 


\section{S1 HAMILTONIAN AND DERIVATION OF DYNAMIC EQUATION EQ. (1)}

In the minimal-coupling scheme, the total Hamiltonian of a molecule in the SPPs environment can be described as ${ }^{1}$

$$
\begin{aligned}
\hat{H}_{\mathrm{tot}}= & \int d \boldsymbol{r} \int_{0}^{\infty} d \omega \hbar \omega \hat{\boldsymbol{f}}^{\dagger}(\boldsymbol{r}, \omega) \cdot \hat{\boldsymbol{f}}(\boldsymbol{r}, \omega)+\sum_{\alpha} \frac{1}{2 m_{\alpha}}\left|\hat{\boldsymbol{p}}_{\alpha}-q_{\alpha} \hat{\boldsymbol{A}}\left(\hat{\boldsymbol{r}}_{\alpha}\right)\right|^{2} \\
& +\frac{1}{2} \int d \boldsymbol{r} \hat{\rho}_{\mathrm{M}}(\boldsymbol{r}) \hat{\phi}_{\mathrm{M}}(\boldsymbol{r})+\int d \boldsymbol{r} \hat{\rho}_{\mathrm{M}}(\boldsymbol{r}) \hat{\phi}(\boldsymbol{r}),
\end{aligned}
$$

where the first term is the Hamiltonian of SPPs, the second term is the kinetic energy of charges in the molecule, the third term is the Coulomb interaction of the molecule, and the fourth term is the Coulomb interaction between the molecule and the SPPs. The details of the four terms will be explained in the following.

In the first term, $\hat{\boldsymbol{f}}^{\dagger}(\boldsymbol{r}, \omega)$ and $\hat{\boldsymbol{f}}(\boldsymbol{r}, \omega)$ are creation and annihilation operator for SPPs, respectively, i.e., $\hat{f}_{i}(\boldsymbol{r}, \omega)\left|\left\{1_{i}(\boldsymbol{r}, \omega)\right\}\right\rangle=|\{0\}\rangle$ and $\hat{f}_{i}^{\dagger}(\boldsymbol{r}, \omega)|\{0\}\rangle=\left|\left\{1_{i}(\boldsymbol{r}, \omega)\right\}\right\rangle$, where $|\{0\}\rangle$ is a vacuum state and $\left|\left\{1_{i}(\boldsymbol{r}, \omega)\right\}\right\rangle$ is a one-polariton state associated with a certain frequency $\omega$ and a polarization direction $i$ at position $\boldsymbol{r}$. The components of the two operators satisfy the commutation relation, ${ }^{2}\left[\hat{f}_{i}(\boldsymbol{r}, \omega), \hat{f}_{j}^{\dagger}\left(\boldsymbol{r}^{\prime}, \omega^{\prime}\right)\right]=\delta_{i j} \delta\left(\boldsymbol{r}-\boldsymbol{r}^{\prime}\right) \delta\left(\omega-\omega^{\prime}\right)$, where $\delta_{i j}$ is a Kronecker delta function and the subscript $i$ (or $j$ ) indicates the spatial coordinate $x, y, z$.

In the second term, $m_{\alpha}$ and $q_{\alpha}$ are the mass of charge and the point charge, respectively. $\hat{\boldsymbol{p}}_{\alpha}$ and $\hat{\boldsymbol{r}}_{\alpha}$ are the momentum and position operators of the charges, respectively. $\hat{\boldsymbol{A}}\left(\hat{\boldsymbol{r}}_{\alpha}\right)$ is the vector potential of the electromagnetic field, which is related to the $\hat{\boldsymbol{E}}(\boldsymbol{r}, \omega)$ operator,

$$
\begin{aligned}
& \hat{\boldsymbol{A}}\left(\hat{\boldsymbol{r}}_{\alpha}\right)=\int_{0}^{\infty} d \omega \frac{\hat{\boldsymbol{E}}^{\perp}\left(\hat{\boldsymbol{r}}_{\alpha}, \omega\right)}{i \omega}+\text { H.c., } \\
& \hat{\boldsymbol{E}}^{\perp(\|)}\left(\hat{\boldsymbol{r}}_{\alpha}, \omega\right)=\int d \boldsymbol{r} \delta^{\perp(\|)}\left(\hat{\boldsymbol{r}}_{\alpha}-\boldsymbol{r}\right) \cdot \hat{\boldsymbol{E}}(\boldsymbol{r}, \omega), \\
& \hat{\boldsymbol{E}}(\boldsymbol{r}, \omega)=i \sqrt{\frac{\hbar}{\pi \epsilon_{0}}} \frac{\omega^{2}}{c^{2}} \int d \boldsymbol{r}^{\prime} \sqrt{\epsilon_{\mathrm{I}}(\boldsymbol{r}, \omega)} \overline{\overline{\boldsymbol{G}}}\left(\boldsymbol{r}, \boldsymbol{r}^{\prime}, \omega\right) \cdot \hat{\boldsymbol{f}}\left(\boldsymbol{r}^{\prime}, \omega\right),
\end{aligned}
$$

where $\delta^{\|}(\boldsymbol{r})$ and $\delta^{\perp}(\boldsymbol{r})$ are the longitudinal and the transverse delta functions, respectively. 
$\epsilon_{\mathrm{I}}(\boldsymbol{r}, \omega)$ is the imaginary part of the dielectric function (relative permittivity) $\epsilon(\boldsymbol{r}, \omega)$ of the dielectric environment, $c$ is the speed of light in a vacuum, $\epsilon_{0}$ is the permittivity of free space, and $\overline{\overline{\boldsymbol{G}}}\left(\boldsymbol{r}_{\mathrm{M}}, \boldsymbol{r}, \omega\right)$ follows

$$
\left(\frac{\epsilon\left(\mathbf{r}_{\mathrm{M}}, \omega\right) \omega^{2}}{c^{2}}-\nabla \times \nabla \times\right) \overline{\overline{\boldsymbol{G}}}\left(\boldsymbol{r}_{\mathrm{M}}, \boldsymbol{r}, \omega\right)=-\boldsymbol{\delta}\left(\mathbf{r}_{\mathrm{M}}-\mathbf{r}\right)
$$

In the third term, $\hat{\phi}_{\mathrm{M}}(\boldsymbol{r})$ and $\hat{\rho}_{\mathrm{M}}(\boldsymbol{r})$ are the electric potential operator and the charge density operator of the nuclei and electrons (point charges),

$$
\begin{aligned}
& \hat{\phi}_{\mathrm{M}}(\boldsymbol{r})=\int d \boldsymbol{r}^{\prime} \frac{\hat{\rho}_{\mathrm{M}}\left(\boldsymbol{r}^{\prime}\right)}{4 \pi \epsilon_{0}\left|\boldsymbol{r}-\boldsymbol{r}^{\prime}\right|} \\
& \hat{\rho}_{\mathrm{M}}(\boldsymbol{r})=\sum_{\alpha} q_{\alpha} \delta\left(\boldsymbol{r}-\hat{\boldsymbol{r}}_{\alpha}\right)
\end{aligned}
$$

In the fourth term of Eq. (S1), $\hat{\phi}(\boldsymbol{r})$ is the electric potential operator of the medium, which obeys the Maxwell equation,

$$
\nabla \hat{\phi}(\boldsymbol{r})=-\hat{\boldsymbol{E}}^{\|}(\boldsymbol{r})
$$

where operator $\hat{\boldsymbol{E}}^{\|}(\boldsymbol{r})$ is the summation of all polariton frequency of the operator $\hat{\boldsymbol{E}}^{\|}(\hat{\boldsymbol{r}}, \omega)$ in Eq. (S3),

$$
\hat{\boldsymbol{E}}^{\|}(\boldsymbol{r})=\int d \omega \hat{\boldsymbol{E}}^{\|}(\hat{\boldsymbol{r}}, \omega)+\text { H.c.. }
$$

For simplification of $\hat{H}_{\text {tot }}$ in the Coulomb gauge $\left(\left[\hat{\boldsymbol{A}}\left(\hat{\boldsymbol{r}}_{\alpha}\right), \hat{\boldsymbol{p}}_{\alpha}\right]=0\right)$, we rearrange Eq. (S1) 
as follows,

$$
\begin{aligned}
\hat{H}_{\mathrm{tot}} & =\hat{H}_{\mathrm{mol}}+\hat{H}_{\mathrm{pol}}+\hat{H}_{\mathrm{int}}, \\
\hat{H}_{\mathrm{mol}} & =\sum_{\alpha} \frac{\hat{\boldsymbol{p}}_{\alpha}^{2}}{2 m_{\alpha}}+\frac{1}{2} \int d \boldsymbol{r} \hat{\rho}_{\mathrm{M}}(\boldsymbol{r}) \hat{\phi}_{\mathrm{M}}(\boldsymbol{r}) \\
& =\sum_{\alpha} \frac{\hat{\boldsymbol{p}}_{\alpha}^{2}}{2 m_{\alpha}}+\frac{1}{2} \sum_{\alpha, \beta} \frac{q_{\alpha} q_{\beta}}{4 \pi \epsilon_{0}\left|\hat{\boldsymbol{r}}_{\alpha}-\hat{\boldsymbol{r}}_{\beta}\right|}, \\
\hat{H}_{\mathrm{pol}} & =\int d \boldsymbol{r} \int_{0}^{\infty} \hbar \omega \hat{\boldsymbol{f}}^{\dagger}(\boldsymbol{r}, \omega) \cdot \hat{\boldsymbol{f}}(\boldsymbol{r}, \omega), \\
\hat{H}_{\mathrm{int}} & =-\sum_{\alpha} \frac{q_{\alpha}}{m_{\alpha}} \hat{\boldsymbol{p}}_{\alpha} \cdot \hat{\boldsymbol{A}}\left(\hat{\boldsymbol{r}}_{\alpha}\right)+\sum_{\alpha} \frac{q_{\alpha}^{2}}{2 m_{\alpha}} \hat{\boldsymbol{A}}^{2}\left(\hat{\boldsymbol{r}}_{\alpha}\right)+\int d \boldsymbol{r} \hat{\rho}_{\mathrm{M}}(\boldsymbol{r}) \hat{\phi}(\boldsymbol{r}),
\end{aligned}
$$

where $\hat{H}_{\text {mol }}$ is the molecular Hamiltonian, $\hat{\phi}_{\mathrm{M}}(\boldsymbol{r})$ and $\hat{\rho}_{\mathrm{M}}(\boldsymbol{r})$ are the electric potential operator and the charge density operator of the molecule, respectively, and the subscript $\alpha(\beta)$ is the index of charges composing the molecule. To simplify $\hat{H}_{\text {mol }}$, we apply the Born-Oppenheimer approximation and consider two electronic states with $n$ harmonic vibrational modes. Based on these assumptions, the exact molecular Hamiltonian $\hat{H}_{\mathrm{mol}}$ in Eq. (S11) reduces to,

$$
\hat{H}_{\mathrm{mol}} \approx|\mathrm{g}\rangle\left(\sum_{w=1}^{n} \hbar \omega_{\mathrm{v}, w}\left[b_{w}^{\dagger} b_{w}-\alpha_{w}\left(b_{w}^{\dagger}+b_{w}\right)+\alpha_{w}^{2}\right]\right)\langle\mathrm{g}|+| \mathrm{e}\rangle\left(\hbar \omega_{\mathrm{eg}}+\sum_{w=1}^{n} \hbar \omega_{\mathrm{v}, w} b_{w}^{\dagger} b_{w}\right)\langle\mathrm{e}|
$$

where $\hbar \omega_{\text {eg }}$ is the adiabatic excitation energy (energy gap) between the electronically ground state $|\mathrm{g}\rangle$ and the electronically excited state $|\mathrm{e}\rangle . \omega_{\mathrm{v}, w}$ is the vibrational frequency of the $w$-th mode. $b_{w}$ and $b_{w}^{+}$are the annihilation operator and the creation operator of the $w$-th vibrational mode, respectively. Furthermore, $\left|M_{w}\left(\alpha_{w}\right)\right\rangle$ is the vibrational state (the displaced Fock state) associated with a quanta $M_{w}$ and the Huang-Rhys factor $\alpha_{w}$, which satisfies $\left[b_{w}^{\dagger} b_{w}-\sqrt{\alpha_{w}}\left(b_{w}^{\dagger}+b_{w}\right)+\alpha_{w}\right]\left|M_{w}\left(\alpha_{w}\right)\right\rangle=M_{w}\left|M_{w}\left(\alpha_{w}\right)\right\rangle$.

As for the interaction Hamiltonian $\hat{H}_{\text {int }}$, we drop the $\hat{\boldsymbol{A}}^{2}\left(\hat{\boldsymbol{r}}_{\alpha}\right)$ in Eq. (S13) due to its zero 
contribution to the molecular fluorescence processes. ${ }^{3}$ Therefore, $\hat{H}_{\text {int }}$ becomes

$$
\hat{H}_{\mathrm{int}}=-\sum_{\alpha} \frac{q_{\alpha}}{m_{\alpha}} \hat{\boldsymbol{p}}_{\alpha} \cdot \hat{\boldsymbol{A}}\left(\hat{\boldsymbol{r}}_{\alpha}\right)+\int d \boldsymbol{r} \hat{\rho}_{\mathrm{M}}(\boldsymbol{r}) \hat{\phi}(\boldsymbol{r})
$$

The first term in the above equation can be simplified with the dipole operator of the molecule $\hat{\boldsymbol{\mu}}_{\mathrm{M}} \equiv \sum_{\beta} q_{\beta}\left(\hat{\boldsymbol{r}}_{\beta}-\boldsymbol{r}_{\mathrm{M}}\right)$, where $\boldsymbol{r}_{\mathrm{M}}$ is the position of the molecule. The approximation of the first term in Eq. (S15) is as follows,

$$
\begin{aligned}
-\sum_{\alpha} \frac{q_{\alpha}}{m_{\alpha}} \hat{\boldsymbol{p}}_{\alpha} \cdot \hat{\boldsymbol{A}}\left(\hat{\boldsymbol{r}}_{\alpha}\right) & =-\sum_{\alpha} \frac{q_{\alpha}}{i \hbar}\left[\hat{\boldsymbol{r}}_{\alpha}, \hat{H}_{\mathrm{mol}}\right] \cdot \hat{\boldsymbol{A}}\left(\hat{\boldsymbol{r}}_{\alpha}\right) \\
& \approx-\sum_{\alpha} \frac{q_{\alpha}}{i \hbar}\left[\hat{\boldsymbol{r}}_{\alpha}, \hat{H}_{\mathrm{mol}}\right] \cdot \hat{\boldsymbol{A}}\left(\boldsymbol{r}_{\mathrm{M}}\right) \\
& =-\sum_{\alpha} \frac{q_{\alpha}}{i \hbar}\left[\hat{\boldsymbol{r}}_{\alpha}-\boldsymbol{r}_{\mathrm{M}}, \hat{H}_{\mathrm{mol}}\right] \cdot \hat{\boldsymbol{A}}\left(\boldsymbol{r}_{\mathrm{M}}\right) \\
& =-\frac{1}{i \hbar}\left[\hat{\boldsymbol{\mu}}_{\mathrm{M}}, \hat{H}_{\mathrm{mol}}\right] \cdot \hat{\boldsymbol{A}}\left(\boldsymbol{r}_{\mathrm{M}}\right) .
\end{aligned}
$$

Note that the canonical commutation relation $\left[\hat{\boldsymbol{r}}_{\alpha}, \hat{\boldsymbol{p}}_{\alpha}\right]=i \hbar$ is used in the derivation of Eq. (S16); the electric-dipole approximation $\hat{\boldsymbol{A}}\left(\hat{\boldsymbol{r}}_{\alpha}\right) \approx \hat{\boldsymbol{A}}\left(\boldsymbol{r}_{\mathrm{M}}\right)$ leads to Eq. (S17); the charge neutrality condition $\boldsymbol{r}_{\mathrm{M}} \sum_{\alpha} q_{\alpha}=0$ results in Eq. (S18).

Moreover, by inserting $\hat{I}_{\mathrm{vib}}=\sum_{P_{1} \cdots P_{n}=0}^{\infty} \prod_{w=1}^{n}\left|P_{w}(0)\right\rangle\left\langle P_{w}(0)\right|$ into the electronically excited state in Eq. (S14), and by substituting $\hat{I}_{\mathrm{vib}}=\sum_{M_{1} \cdots M_{n}=0}^{\infty} \prod_{w=1}^{n}\left|M_{w}\left(\alpha_{M}\right)\right\rangle\left\langle M_{w}\left(\alpha_{w}\right)\right|$ into the electronically ground state in Eq. (S14), we derive an alternative expression of $\hat{H}_{\text {mol }}$ in Eq. (S19),

$$
\begin{aligned}
\hat{H}_{\mathrm{mol}}= & \sum_{M_{1} \cdots M_{n}=0}^{\infty}|\mathrm{g}\rangle\left\langle\mathrm{g}\left|\otimes\left(\sum_{w=1}^{n} \hbar \omega_{\mathrm{v}, w} M_{w}\right) \prod_{w=1}^{n}\right| M_{w}\left(\alpha_{w}\right)\right\rangle\left\langle M_{w}\left(\alpha_{w}\right)\right| \\
& +\sum_{P_{1} \cdots P_{n}=0}^{\infty}|\mathrm{e}\rangle\left\langle\mathrm{e}\left|\otimes\left(\hbar \omega_{\mathrm{eg}}+\sum_{w=1}^{n} \hbar \omega_{\mathrm{v}, w} P_{w}\right) \prod_{w=1}^{n}\right| P_{w}(0)\right\rangle\left\langle P_{w}(0)\right|,
\end{aligned}
$$

where $\left|P_{w}(0)\right\rangle$ is the vibrational state (the Fock state) associated with a quanta $P_{w}$ and the Huang-Rhys factor $\alpha_{w}=0$. If we consider a molecule with a negligible permanent 
dipole moment $\left(\left\langle\mathrm{e}\left|\hat{\boldsymbol{\mu}}_{\mathrm{M}}\right| \mathrm{e}\right\rangle=\left\langle\mathrm{g}\left|\hat{\boldsymbol{\mu}}_{\mathrm{M}}\right| \mathrm{g}\right\rangle \approx 0\right)$ and an appreciable transition dipole moment $\left\langle\mathrm{e}\left|\hat{\boldsymbol{\mu}}_{\mathrm{M}}\right| \mathrm{g}\right\rangle=\left\langle\mathrm{g}\left|\hat{\boldsymbol{\mu}}_{\mathrm{M}}\right| \mathrm{e}\right\rangle=\boldsymbol{\mu}$, the first term in Eq. (S19) can be further reduced to

$$
\begin{aligned}
& -\frac{\left[\hat{\boldsymbol{\mu}}_{\mathrm{M}}, \hat{H}_{\text {molecule }}\right] \cdot \hat{\boldsymbol{A}}\left(\boldsymbol{r}_{\mathrm{M}}\right)}{i \hbar}=\frac{i}{\hbar}\left(\hat{I} \hat{\boldsymbol{\mu}}_{\mathrm{M}} \hat{H}_{\text {molecule }}-\hat{H}_{\text {molecule }} \hat{\boldsymbol{\mu}}_{\mathrm{M}} \hat{I}\right) \cdot \hat{\boldsymbol{A}}\left(\boldsymbol{r}_{\mathrm{M}}\right) \\
& =\frac{i}{\hbar}\left\{| \mathrm { g } \rangle \langle \mathrm { e } | \boldsymbol { \mu } \left[\hbar \omega_{\mathrm{eg}}+\sum_{P_{1} \cdots P_{n}}^{\infty} \sum_{w=1}^{n} \hbar \omega_{\mathrm{v}, w} P_{w}\left(\prod_{w=1}^{n}\left|P_{w}(0)\right\rangle\left\langle P_{w}(0)\right|\right.\right.\right. \\
& \left.\left.\left.\quad-\prod_{w=1}^{n}\left|P_{w}\left(\alpha_{w}\right)\right\rangle\left\langle P_{w}\left(\alpha_{w}\right)\right|\right)\right]-H . c\right\} \cdot \hat{\boldsymbol{A}}\left(\boldsymbol{r}_{\mathrm{A}}\right) \\
& \approx i\left\{|\mathrm{~g}\rangle\langle\mathrm{e}| \boldsymbol{\mu} \omega_{\mathrm{eg}}-\mathrm{H} . \mathrm{c}\right\} \cdot \hat{\boldsymbol{A}}\left(\boldsymbol{r}_{\mathrm{A}}\right) \\
& =\{|\mathrm{g}\rangle\langle\mathrm{e}| \boldsymbol{\mu}-H . c\} \cdot\left\{\int_{0}^{\infty} d \omega \frac{\omega_{\mathrm{eg}}}{\omega} \hat{\boldsymbol{E}}^{\perp}\left(\boldsymbol{r}_{\mathrm{A}}, \omega\right)-\mathrm{H} . \mathrm{c}\right\} \\
& \approx-\left\{|\mathrm{g}\rangle\left\langle\mathrm{e}\left|\boldsymbol{\mu} \cdot \hat{\boldsymbol{E}}^{\perp(-)}\left(\boldsymbol{r}_{\mathrm{A}}\right)+\right| \mathrm{e}\right\rangle\langle\mathrm{g}| \boldsymbol{\mu} \cdot \hat{\boldsymbol{E}}^{\perp(+)}\left(\boldsymbol{r}_{\mathrm{A}}\right)\right\}
\end{aligned}
$$

where the identity operator of the molecular system $\hat{I}$ is used:

$$
\begin{aligned}
\hat{I} & =\hat{I}_{\text {ele }} \otimes \hat{I}_{\mathrm{vib}}=(|\mathrm{e}\rangle\langle\mathrm{e}|+| \mathrm{g}\rangle\langle\mathrm{g}|) \otimes \sum_{M_{1} \cdots M_{n}=0}^{\infty} \prod_{w=1}^{n}\left|M_{w}(0)\right\rangle\left\langle M_{w}(0)\right| \\
& =|\mathrm{e}\rangle\left\langle\mathrm{e}\left|\otimes \sum_{M_{1} \cdots M_{n}=0}^{\infty} \prod_{w=1}^{n}\right| M_{w}\left(\alpha_{w}\right)\right\rangle\left\langle M_{w}\left(\alpha_{w}\right)|+| \mathrm{g}\right\rangle\left\langle\mathrm{g}\left|\otimes \sum_{P_{1} \cdots P_{n}=0}^{\infty} \prod_{w=1}^{n}\right| P_{w}(0)\right\rangle\left\langle P_{w}(0)\right|
\end{aligned}
$$

Also, to derive Eq. (S22), we implicitly apply the Condon approximation so that the transition dipole moment of the molecule is independent of its nuclear coordinates, which is shown 
below

$$
\begin{aligned}
& \sum_{P_{1} \cdots P_{n}=0}^{\infty}\left(\sum_{w=1}^{n} \hbar \omega_{\mathrm{v}, w} P_{w}\right)\left(\prod_{w=1}^{n}\left|P_{w}(0)\right\rangle\left\langle P_{w}(0)\left|-\prod_{w=1}^{n}\right| P_{w}\left(\alpha_{w}\right)\right\rangle\left\langle P_{w}\left(\alpha_{w}\right)\right|\right) \\
= & \sum_{w=1}^{n} \sum_{P_{w}=0}^{\infty} \hbar \omega_{\mathrm{v}, w} P_{w} \sum_{\substack{P_{1} \cdots P_{w-1} \\
P_{w+1} \cdots P_{n}=0}}^{\infty}\left(\prod_{w=1}^{n}\left|P_{w}(0)\right\rangle\left\langle P_{w}(0)\left|\prod_{w^{\prime} \neq w}^{n}\right| P_{w^{\prime}}(0)\right\rangle\left\langle P_{w^{\prime}}(0)\right|\right. \\
& \left.-\prod_{w=1}^{n}\left|P_{w}\left(\alpha_{w}\right)\right\rangle\left\langle P_{w}\left(\alpha_{w}\right)\left|\prod_{w^{\prime} \neq w}^{n}\right| P_{w^{\prime}}\left(\alpha_{w^{\prime}}\right)\right\rangle\left\langle P_{w^{\prime}}\left(\alpha_{w^{\prime}}\right)\right|\right) \\
= & \sum_{w=1}^{n} \sum_{P_{w}=0}^{\infty} \hbar \omega_{\mathrm{v}, w} P_{w}\left(\left|P_{w}(0)\right\rangle\left\langle P_{w}(0)|-| P_{w}\left(\alpha_{w}\right)\right\rangle\left\langle P_{w}\left(\alpha_{w}\right)\right|\right) \\
= & \sum_{w=1}^{n} \sum_{P_{w}=0}^{\infty} \hbar \omega_{\mathrm{v}, w}\left(b_{w}^{\dagger} b_{w}\left|P_{w}(0)\right\rangle\left\langle P_{w}(0)\left|-\bar{b}_{w}^{\dagger} \bar{b}_{w}\right| P_{w}\left(\alpha_{w}\right)\right\rangle\left\langle P_{w}\left(\alpha_{w}\right)\right|\right) \approx 0,
\end{aligned}
$$

where $\bar{b}_{w}^{\dagger}=b_{w}^{\dagger}-\sqrt{\alpha_{w}}$. In order to derive Eq. (S24), $\omega=\omega_{\mathrm{eg}}$ is set in the integral due to the pre-trace rotating-wave approximation. ${ }^{4}$

To deal with the second term in Eq. (S15), $\hat{\rho}_{\mathrm{M}}(\boldsymbol{r})$ is expanded in a multi-polar form and we only retain the first non-vanishing term,

$$
\begin{aligned}
\hat{\rho}_{\mathrm{M}}(\boldsymbol{r}) & \approx \sum_{\alpha} q_{\alpha} \delta\left(\boldsymbol{r}-\boldsymbol{r}_{\mathrm{M}}\right)-\nabla \cdot\left(\delta\left(\boldsymbol{r}-\boldsymbol{r}_{\mathrm{M}}\right) \sum_{\alpha} q_{\alpha}\left(\hat{\boldsymbol{r}}_{\boldsymbol{\alpha}}-\boldsymbol{r}_{\mathrm{M}}\right)\right) \\
& =-\nabla \cdot \delta\left(\boldsymbol{r}-\boldsymbol{r}_{\mathrm{M}}\right) \hat{\boldsymbol{\mu}}_{\mathrm{M}} .
\end{aligned}
$$

Plugging Eq. (S29) into Eq. (S15), we get

$$
\begin{aligned}
& \int d \boldsymbol{r} \hat{\rho}_{\mathrm{M}}(\boldsymbol{r}) \hat{\phi}(\boldsymbol{r})=-\int d \boldsymbol{r}\left(\nabla \cdot \delta\left(\boldsymbol{r}-\boldsymbol{r}_{\mathrm{M}}\right) \hat{\boldsymbol{\mu}}_{\mathrm{M}}\right) \hat{\phi}(\boldsymbol{r}) \\
& =\int d \boldsymbol{r}\left(\delta\left(\boldsymbol{r}-\boldsymbol{r}_{\mathrm{M}}\right) \hat{\boldsymbol{\mu}}_{\mathrm{M}}\right) \cdot \nabla \hat{\phi}(\boldsymbol{r})=\hat{\boldsymbol{\mu}}_{\mathrm{M}} \cdot \nabla \hat{\phi}\left(\boldsymbol{r}_{\mathrm{M}}\right) \\
& =-\hat{\boldsymbol{\mu}}_{\mathrm{M}} \cdot \hat{\boldsymbol{E}}^{\|}\left(\boldsymbol{r}_{\mathrm{M}}\right)=-\hat{I} \hat{\boldsymbol{\mu}}_{\mathrm{M}} \hat{I} \cdot \hat{\boldsymbol{E}}^{\|}\left(\boldsymbol{r}_{\mathrm{M}}\right) \\
& =-|\mathrm{e}\rangle\left\langle\mathrm{g}\left|\boldsymbol{\mu} \cdot \hat{\boldsymbol{E}}^{\|(+)}\left(\boldsymbol{r}_{\mathrm{M}}\right)-\right| \mathrm{g}\right\rangle\langle\mathrm{e}| \boldsymbol{\mu} \cdot \hat{\boldsymbol{E}}^{\|(-)}\left(\boldsymbol{r}_{\mathrm{M}}\right),
\end{aligned}
$$

where integration by parts is used to derive Eq. (S30), and the rotating-wave approximation 
is applied to derive Eq. (S32).

By combining Eq. (S32) and Eq. (S24), we get the approximated interaction Hamiltonian,

$$
\hat{H}_{\mathrm{int}}=-\left(|\mathrm{e}\rangle\left\langle\mathrm{g}\left|\hat{\boldsymbol{E}}^{(+)}\left(\boldsymbol{r}_{\mathrm{M}}\right) \cdot \boldsymbol{\mu}+\right| \mathrm{g}\right\rangle\langle\mathrm{e}| \hat{\boldsymbol{E}}^{(-)}\left(\boldsymbol{r}_{\mathrm{M}}\right) \cdot \boldsymbol{\mu}\right),
$$

where the property of $\hat{\boldsymbol{E}}^{(+)}\left(\boldsymbol{r}_{\mathrm{M}}\right)$ and the Helmholtz decomposition theorem are applied, ${ }^{1}$

$$
\begin{aligned}
& \hat{\boldsymbol{E}}\left(\boldsymbol{r}_{\mathrm{M}}\right)=\hat{\boldsymbol{E}}^{(+)}\left(\boldsymbol{r}_{\mathrm{M}}\right)+\text { H.c. } \\
& \hat{\boldsymbol{E}}^{( \pm)}\left(\boldsymbol{r}_{\mathrm{M}}\right)=\hat{\boldsymbol{E}}^{\|( \pm)}\left(\boldsymbol{r}_{\mathrm{M}}\right)+\hat{\boldsymbol{E}}^{\perp( \pm)}\left(\boldsymbol{r}_{\mathrm{M}}\right) .
\end{aligned}
$$

In summary, the Hamiltonian including a molecular system, polariton field (or dressed photon field), and their interactions is expressed as

$$
\begin{aligned}
\hat{H}= & |\mathrm{g}\rangle \sum_{w=1}^{N}\left(\hbar \omega_{\mathrm{v}, w}\left[b_{w}^{\dagger} b_{w}-\alpha_{w}\left(b_{w}^{\dagger}+b_{w}\right)+\alpha_{w}^{2}\right]\right)\langle\mathrm{g}|+| \mathrm{e}\rangle\left(\hbar \omega_{\mathrm{eg}}+\sum_{w=1}^{N} \hbar \omega_{\mathrm{v}, w} b_{w}^{\dagger} b_{w}\right)\langle\mathrm{e}| \\
& +\int d \boldsymbol{r} \int_{0}^{\infty} d \omega \hbar \omega \hat{f}^{\dagger}(\boldsymbol{r}, \omega) \cdot \hat{\boldsymbol{f}}(\boldsymbol{r}, \omega)-\left[|\mathrm{e}\rangle\left\langle\mathrm{g}\left|\hat{\boldsymbol{E}}^{(+)}\left(\boldsymbol{r}_{\mathrm{A}}\right) \cdot \boldsymbol{\mu}+\right| \mathrm{g}\right\rangle\langle\mathrm{e}| \hat{\boldsymbol{E}}^{(-)}\left(\boldsymbol{r}_{\mathrm{A}}\right) \cdot \boldsymbol{\mu}\right] .
\end{aligned}
$$

In order to study the quantum dynamics of the proposed Hamiltonian Eq. (S36), we apply the Wigner-Weisskopf theory. In this theory, the wavefunction ansatz $|\psi(t)\rangle$ can be chosen as

$$
\begin{aligned}
|\psi(t)\rangle= & \sum_{M_{1}, \cdots, M_{n}=0}^{\infty} C_{M_{1}, \cdots, M_{n}}^{\mathrm{ex}}(t) e^{-i\left(\omega_{\mathrm{eg}}+\sum_{w=1}^{n} \omega_{\mathrm{v}, w} M_{w}\right) t}|\mathrm{e}\rangle|\{0\}\rangle \prod_{w=1}^{n}\left|M_{w}(0)\right\rangle \\
& +\sum_{P_{1}, \cdots, P_{n}=0}^{\infty} \int d \boldsymbol{r} \int_{0}^{\infty} d \omega C_{P_{1}, \cdots, P_{n}}^{\mathrm{gi}}(\boldsymbol{r}, \omega, t) e^{-i\left(\omega+\sum_{w=1}^{n} \omega_{\mathrm{v}, w} P_{w}\right) t}|\mathrm{~g}\rangle\left|\left\{1_{\mathrm{i}}(\boldsymbol{r}, \omega)\right\}\right\rangle \prod_{w=1}^{n}\left|P_{w}\left(\alpha_{w}\right)\right\rangle,
\end{aligned}
$$

where $C_{M_{1}, \cdots, M_{n}}^{\mathrm{ex}}(t)$ and $C_{P_{1}, \cdots, P_{n}}^{\mathrm{gi}}(\boldsymbol{r}, \omega, t)$ are the coefficients of molecular electronically excited state (denoted by the superscript ex) with the quanta of $n$ different vibrational modes 
(denoted by the sub-script $K_{1}, K_{2}, \cdots, K_{n}$ ) and the coefficients of molecular electronically ground state (denoted by the superscript g) with the polarization direction i having the quanta of $n$ different vibrational modes (denoted by the sub-script $P_{1}, P_{2}, \cdots, P_{n}$ ), respectively. Plugging the wavefunction ansatz $|\psi(t)\rangle$ into the time-dependent Schrödinger equation $i \hbar \partial|\psi(t)\rangle / \partial t=\hat{H}|\psi(t)\rangle$, the left-hand side becomes

$$
\begin{aligned}
& i \hbar \partial|\psi(t)\rangle / \partial t= \\
& \sum_{M_{1}, \cdots, M_{n}=0}^{\infty}\left\{i \hbar \dot{C}_{M_{1}, \cdots, M_{n}}^{\mathrm{ex}}(t)+\hbar\left(\omega_{\mathrm{eg}}+\sum_{w=1}^{n} \omega_{\mathrm{v}, w} M_{w}\right) C_{M_{1}, \cdots, M_{n}}^{\mathrm{ex}}(t)\right\} e^{-i\left(\omega_{\mathrm{eg}}+\sum_{w=1}^{n} \omega_{\mathrm{v}, w} M_{w}\right) t} \\
& |\mathrm{e}\rangle|\{0\}\rangle \prod_{w=1}^{n}\left|M_{w}(0)\right\rangle+\sum_{P_{1}, \cdots, P_{n}=0}^{\infty} \int d \boldsymbol{r} \int_{0}^{\infty} d \omega\left\{i \hbar \dot{C}_{P_{1}, \cdots, P_{n}}^{\mathrm{gi}}(\boldsymbol{r}, \omega, t)+\hbar\left(\omega+\sum_{w=1}^{n} \omega_{\mathrm{v}, w} P_{w}\right)\right. \\
& \left.C_{P_{1}, \cdots, P_{n}}^{\mathrm{gi}}(\boldsymbol{r}, \omega, t)\right\} e^{-i\left(\omega+\sum_{w=1}^{n} \omega_{\mathrm{v}, w} P_{w}\right) t}|\mathrm{~g}\rangle\left|\left\{1_{i}(\boldsymbol{r}, \omega)\right\}\right\rangle \prod_{w=1}^{n}\left|P_{w}\left(\alpha_{w}\right)\right\rangle,
\end{aligned}
$$

For the right-hand side of the time-dependent Schrödinger equation, $\hat{H}_{\text {tot }}|\psi(t)\rangle$ can be separated into three parts: $\hat{H}_{\text {mol }}|\psi(t)\rangle ; \hat{H}_{\text {pol }}|\psi(t)\rangle ; \hat{H}_{\text {int }}|\psi(t)\rangle$, and each of them is evaluated as follows,

$$
\begin{aligned}
& \hat{H}_{\mathrm{mol}}|\psi(t)\rangle \\
& =\sum_{M_{1}, \cdots, M_{n}=0}^{\infty}\left(\hbar \omega_{\mathrm{eg}}+\sum_{w=1}^{n} \hbar \omega_{\mathrm{v}, w} M_{w}\right) C_{M_{1}, \cdots, M_{n}}^{\mathrm{ex}}(t) e^{-i\left(\omega_{\mathrm{eg}}+\sum_{w=1}^{n} \omega_{\mathrm{v}, w} M_{w}\right) t}|\mathrm{e}\rangle|\{0\}\rangle \prod_{w=1}^{n}\left|M_{w}(0)\right\rangle \\
& +\sum_{P_{1}, \cdots, P_{n}=0}^{\infty} \int d \boldsymbol{r} \int_{0}^{\infty} d \omega \sum_{w=1}^{n}\left(\hbar \omega_{\mathrm{v}, w} P_{w}\right) C_{P_{1}, \cdots, P_{n}}^{\mathrm{gi}}(\boldsymbol{r}, \omega, t) e^{-i\left(\omega+\sum_{w=1}^{n} \omega_{\mathrm{v}, w} P_{w}\right) t}|\mathrm{~g}\rangle\left|\left\{1_{i}(\boldsymbol{r}, \omega)\right\}\right\rangle \prod_{w=1}^{n}\left|P_{w}\left(\alpha_{w}\right)\right\rangle,
\end{aligned}
$$

$$
\begin{aligned}
& \hat{H}_{\mathrm{pol}}|\psi(t)\rangle \\
& =\sum_{P_{1}, \cdots, P_{n}=0}^{\infty} \int d \boldsymbol{r} \int_{0}^{\infty} d \omega(\hbar \omega) C_{P_{1}, \cdots, P_{n}}^{\mathrm{gi}}(\boldsymbol{r}, \omega, t) e^{-i\left(\omega+\sum_{w=1}^{n} \omega_{\mathrm{v}, w} P_{w}\right) t}|\mathrm{~g}\rangle\left|\left\{1_{i}(\boldsymbol{r}, \omega)\right\}\right\rangle \prod_{w=1}^{n}\left|P_{w}\left(\alpha_{w}\right)\right\rangle,
\end{aligned}
$$




$$
\begin{aligned}
& \hat{H}_{\mathrm{int}}|\psi(t)\rangle \\
& =-\sum_{M_{1}, \cdots, M_{n}=0}^{\infty}\left(\hat{E}_{j}^{(-)}\left(\boldsymbol{r}_{\mathrm{M}}\right) \mu_{j}\right) C_{M_{1}, \cdots, M_{n}}^{\mathrm{ex}}(t) e^{-i\left(\omega_{\mathrm{eg}}+\sum_{w=1}^{n} \omega_{\mathrm{v}, w} M_{w}\right) t}|\mathrm{~g}\rangle|\{0\}\rangle \prod_{w=1}^{n}\left|M_{w}(0)\right\rangle \\
& -\sum_{P_{1}, \cdots, P_{n}=0}^{\infty}\left(\hat{E}_{j}^{(+)}\left(\boldsymbol{r}_{\mathrm{M}}\right) \mu_{j}\right) \int d \boldsymbol{r} \int_{0}^{\infty} d \omega C_{P_{1}, \cdots, P_{n}}^{\mathrm{gi}}(\boldsymbol{r}, \omega, t) e^{-i\left(\omega+\sum_{w=1}^{n} \omega_{\mathrm{v}, w} P_{w}\right) t}|\mathrm{e}\rangle\left|\left\{1_{i}(\boldsymbol{r}, \omega)\right\}\right\rangle \prod_{w=1}^{n}\left|P_{w}\left(\alpha_{w}\right)\right\rangle \\
& =-\sum_{M_{1}, \cdots, M_{n}=0}^{\infty} \int_{0}^{\infty} d \omega \mu_{j}(-i) \sqrt{\frac{\hbar}{\pi \epsilon_{0}}} \frac{\omega^{2}}{c^{2}} \int d \boldsymbol{r} \sqrt{\epsilon_{\mathrm{I}}(\boldsymbol{r}, \omega)} G_{j i}^{*}\left(\boldsymbol{r}_{\mathrm{M}}, \boldsymbol{r}, \omega\right) C_{M_{1}, \cdots, M_{n}}^{\mathrm{ex}}(t) e^{-i\left(\omega_{\mathrm{eg}}+\sum_{w=1}^{n} \omega_{\mathrm{v}, w} M_{w}\right) t} \\
& \quad|\mathrm{~g}\rangle\left|\left\{1_{i}(\boldsymbol{r}, \omega)\right\}\right\rangle \prod_{w=1}^{n}\left|M_{w}(0)\right\rangle-\sum_{P_{1}, \cdots, P_{n}=0}^{\infty} \int_{0}^{\infty} d \omega \mu_{j}(i) \sqrt{\frac{\hbar}{\pi \epsilon_{0}}} \frac{\omega^{2}}{c^{2}} \int d \boldsymbol{r} \sqrt{\epsilon_{\mathrm{I}}(\boldsymbol{r}, \omega)} G_{j i}\left(\boldsymbol{r}_{\mathrm{M}}, \boldsymbol{r}, \omega\right) \\
& \quad C_{P_{1}, \cdots, P_{n}}^{\mathrm{gi}}(\boldsymbol{r}, \omega, t) e^{-i\left(\omega+\sum_{w=1}^{n} \omega_{\mathrm{v}, w} P_{w}\right) t}|\mathrm{e}\rangle|\{0\}\rangle \prod_{w=1}^{n}\left|P_{w}\left(\alpha_{w}\right)\right\rangle,
\end{aligned}
$$

where $\hat{f}_{i}^{+}\left(\boldsymbol{r}^{\prime}, \omega^{\prime}\right)|\{0\}\rangle=\left|\left\{1_{i}\left(\boldsymbol{r}^{\prime}, \omega^{\prime}\right)\right\}\right\rangle$ and $\hat{f}_{i}\left(\boldsymbol{r}^{\prime}, \omega^{\prime}\right)\left|\left\{1_{k}(\boldsymbol{r}, \omega)\right\}\right\rangle=\delta\left(\boldsymbol{r}-\boldsymbol{r}^{\prime}\right) \delta\left(\omega-\omega^{\prime}\right) \delta_{i, k}|\{0\}\rangle$ are used. $G_{j i}\left(\boldsymbol{r}_{\mathrm{M}}, \boldsymbol{r}, \omega\right)$ is the matrix element of the dyadic Green's function $\overline{\bar{G}}\left(\boldsymbol{r}_{\mathrm{M}}, \boldsymbol{r}, \omega\right)$. Comparing the right-hand side and the left-hand side of the time-dependent Schrödinger equation and using the notation of an auxiliary dyadic Green's function,

$$
\tilde{\tilde{G}}_{j i}\left(\boldsymbol{r}_{\mathrm{M}}, \boldsymbol{r}, \omega\right)=i \mu_{j} \frac{\omega^{2}}{c^{2}} \sqrt{\frac{\hbar}{\pi \epsilon_{0}}} \sqrt{\epsilon_{\mathrm{I}}(\boldsymbol{r}, \omega)} G_{j i}\left(\boldsymbol{r}_{\mathrm{M}}, \boldsymbol{r}, \omega\right)
$$

we get the following result,

$$
\begin{aligned}
& -i \hbar\left\{\sum_{M_{1}, \cdots, M_{n}=0}^{\infty} \dot{C}_{M_{1}, \cdots, M_{n}}^{\mathrm{ex}}(t) e^{-i\left(\omega_{\mathrm{eg}}+\sum_{w=1}^{n} \omega_{\mathrm{v}, w} M_{w}\right) t}|\mathrm{e}\rangle|\{0\}\rangle \prod_{w=1}^{n}\left|M_{w}(0)\right\rangle\right. \\
& \left.+\sum_{P_{1}, \cdots, P_{n}=0}^{\infty} \int d \boldsymbol{r} \int_{0}^{\infty} d \omega \dot{C}_{P_{1}, \cdots, P_{n}}^{\mathrm{gi}}(\boldsymbol{r}, \omega, t) e^{-i\left(\omega+\sum_{w=1}^{n} \omega_{\mathrm{v}, w} P_{w}\right) t}|\mathrm{~g}\rangle\left|\left\{1_{i}(\boldsymbol{r}, \omega)\right\}\right\rangle \prod_{w=1}^{n}\left|P_{w}\left(\alpha_{w}\right)\right\rangle\right\} \\
& =\int d \omega \int d \boldsymbol{r}\left\{\sum_{M_{1}, \cdots, M_{n}=0}^{\infty} \tilde{\tilde{G}}_{j i}^{*}\left(\boldsymbol{r}_{\mathrm{M}}, \boldsymbol{r}, \omega\right) C_{M_{1}, \cdots, M_{n}}^{\mathrm{ex}}(t) e^{-i\left(\omega_{\mathrm{eg}}+\sum_{w=1}^{n} \omega_{\mathrm{v}, w} M_{w}\right) t}|\mathrm{~g}\rangle\left|\left\{1_{i}(\boldsymbol{r}, \omega)\right\}\right\rangle \prod_{w=1}^{n}\left|M_{w}(0)\right\rangle\right. \\
& \left.+\sum_{P_{1}, \cdots, P_{n}=0}^{\infty} \tilde{\tilde{G}}_{j i}\left(\boldsymbol{r}_{\mathrm{M}}, \boldsymbol{r}, \omega\right) C_{P_{1}, \cdots, P_{n}}^{\mathrm{gi}}(\boldsymbol{r}, \omega, t) e^{-i\left(\omega+\sum_{w=1}^{n} \omega_{\mathrm{v}, w} P_{w}\right) t}|\mathrm{e}\rangle|\{0\}\rangle \prod_{w=1}^{n}\left|P_{w}\left(\alpha_{w}\right)\right\rangle\right\} .
\end{aligned}
$$

Let $\langle\mathrm{e}|\langle\{0\}| \prod_{w=1}^{n}\left\langle K_{w}(0)\right|$ and $\langle\mathrm{g}|\left\langle\left\{1_{i}\left(\boldsymbol{r}^{\prime}, \omega^{\prime}\right)\right\}\right| \prod_{w=1}^{n}\left\langle K_{w}\left(\alpha_{w}\right)\right|$ act on the above equation 
sequentially, we derive

$$
\begin{aligned}
& i \hbar \dot{C}_{K_{1}, \cdots, K_{n}}^{\mathrm{ex}}(t) e^{-i\left(\omega_{\mathrm{eg}}+\sum_{w=1}^{n} \omega_{\mathrm{v}, w} K_{w}\right) t} \\
& =-\int d \omega \int d \boldsymbol{r} \sum_{P_{1}, \cdots, P_{n}=0}^{\infty} \tilde{\tilde{G}}_{j i}\left(\boldsymbol{r}_{\mathrm{M}}, \boldsymbol{r}, \omega\right) \prod_{w=1}^{n}\left\langle K_{w}(0) \mid P_{w}\left(\alpha_{w}\right)\right\rangle C_{P_{1}, \cdots, P_{n}}^{\mathrm{gi}}(\boldsymbol{r}, \omega, t) e^{-i\left(\omega+\sum_{w=1}^{n} \omega_{\mathrm{v}, w} P_{w}\right) t}
\end{aligned}
$$

$$
\begin{aligned}
& i \hbar \dot{C}_{K_{1}, \cdots, K_{n}}^{\mathrm{gi}}(\boldsymbol{r}, \omega, t) e^{-i\left(\omega+\sum_{w=1}^{n} \omega_{\mathrm{v}, w} K_{w}\right) t} \\
& =-\tilde{\tilde{G}}_{j i}^{*}\left(\boldsymbol{r}_{\mathrm{M}}, \boldsymbol{r}, \omega\right) \sum_{M_{1}, \cdots, M_{n}=0}^{\infty} C_{M_{1}, \cdots, M_{n}}^{\mathrm{ex}}(t) e^{-i\left(\omega_{\mathrm{eg}}+\sum_{w=1}^{n} \omega_{\mathrm{v}, w} M_{w}\right) t} \prod_{w=1}^{n}\left\langle K_{w}\left(\alpha_{w}\right) \mid M_{w}(0)\right\rangle
\end{aligned}
$$

With the initial conditions $C_{K_{1}, \cdots, K_{n}}^{\mathrm{gi}}(\boldsymbol{r}, \omega, 0)=0$, Eq. (S45) can be integrated, and the result is

$$
\begin{aligned}
& C_{K_{1}, \cdots, K_{n}}^{\mathrm{gi}}(\boldsymbol{r}, \omega, t) \\
& =\int_{0}^{t} d t^{\prime} \frac{-\tilde{\tilde{G}}_{j i}^{*}\left(\boldsymbol{r}_{\mathrm{M}}, \boldsymbol{r}, \omega\right)}{i \hbar} \sum_{M_{1}, \cdots, M_{n}=0}^{\infty} \prod_{w=1}^{n}\left\langle K_{w}\left(\alpha_{1}\right) \mid M_{w}(0)\right\rangle C_{M_{1}, \cdots, M_{n}}^{\mathrm{ex}}\left(t^{\prime}\right) e^{-i t^{\prime}\left[\left(\omega_{\mathrm{eg}}-\omega\right)+\sum_{w=1}^{n} \omega_{\mathrm{v}, w}\left(M_{w}-K_{w}\right)\right]},
\end{aligned}
$$

substituting this result into Eq. (S44), we can derive the following result,

$$
\begin{aligned}
& \dot{C}_{K_{1}, \cdots, K_{n}}^{\mathrm{ex}}(t) \\
&=\int_{0}^{\infty} d \omega \int_{0}^{t} d t^{\prime} \int d \boldsymbol{r} \frac{\tilde{\tilde{G}}_{j i}\left(\boldsymbol{r}_{\mathrm{M}}, \boldsymbol{r}, \omega\right) \tilde{\tilde{G}}_{k i}^{*}\left(\boldsymbol{r}_{\mathrm{M}}, \boldsymbol{r}, \omega\right)}{-\hbar^{2}} \sum_{P_{1}, \cdots, P_{n}=0}^{\infty}\left\{\sum_{M_{1}, \cdots, M_{n}=0}^{\infty} \prod_{w=1}^{n}\left\langle K_{w}(0) \mid P_{w}\left(\alpha_{w}\right)\right\rangle\right. \\
&\left.\left\langle P_{w}\left(\alpha_{w}\right) \mid M_{w}(0)\right\rangle\right\} e^{-i t\left[\left(\omega-\omega_{\mathrm{eg}}\right)+\sum_{w=1}^{n} \omega_{\mathrm{v}, w}\left(P_{w}-K_{w}\right)\right]} e^{-i t^{\prime}\left[\left(\omega_{\mathrm{eg}}-\omega\right)+\sum_{w=1}^{n} \omega_{\mathrm{v}, w}\left(M_{w}-P_{w}\right)\right]} C_{M_{1}, \cdots, M_{n}}^{\mathrm{ex}}\left(t^{\prime}\right) .
\end{aligned}
$$

Using the relationship, ${ }^{1}$

$$
\operatorname{Im} G_{j k}\left(\boldsymbol{r}_{\mathrm{M}}, \boldsymbol{r}_{\mathrm{B}}, \omega\right)=\int d \boldsymbol{r} \frac{\omega^{2}}{c^{2}} \epsilon_{\mathrm{I}}(\boldsymbol{r}, \omega) G_{j i}\left(\boldsymbol{r}_{\mathrm{M}}, \boldsymbol{r}, \omega\right) G_{k i}^{*}\left(\boldsymbol{r}_{\mathrm{B}}, \boldsymbol{r}, \omega\right)
$$


we obtain Eqs. (1)-(3) in the manuscript,

$$
\begin{aligned}
& \dot{C}_{K_{1}, \cdots, K_{n}}^{\mathrm{ex}}(t)=-\frac{\mu_{j} \mu_{k}}{\hbar \pi \epsilon_{0}} \sum_{L_{1}, \cdots, L_{n}=0}^{\infty} \int_{0}^{t} d t^{\prime}\left\{\int_{0}^{\infty} d \omega \frac{\omega^{2}}{c^{2}} \operatorname{Im} G_{j k}\left(\boldsymbol{r}_{\mathrm{M}}, \boldsymbol{r}_{\mathrm{M}}, \omega\right) e^{-i\left(\omega-\omega_{\mathrm{eg}}\right)\left(t-t^{\prime}\right)}\right\} \\
& \prod_{s=1}^{n}\left\{\sum_{M_{s}=0}^{\infty}\left(\left\langle K_{s}(0) \mid M_{s}\left(\alpha_{s}\right)\right\rangle e^{i t \omega_{\mathrm{v}, s}\left(K_{s}-M_{s}\right)}\left\langle M_{s}\left(\alpha_{s}\right) \mid L_{s}(0)\right\rangle e^{i t^{\prime} \omega_{\mathrm{v}, s}\left(M_{s}-L_{s}\right)}\right)\right\} C_{L_{1}, \cdots, L_{n}}^{\mathrm{ex}}\left(t^{\prime}\right),
\end{aligned}
$$

where $\left\langle K_{s}(0) \mid M_{s}\left(\alpha_{s}\right)\right\rangle$ is the vibrational wavefunction overlap of the $s$-th mode between the electronically excited state and the electronically ground state,

$$
\left\langle K_{s}(0) \mid M_{s}\left(\alpha_{s}\right)\right\rangle=e^{-\frac{\alpha_{s}}{2}} \sqrt{\frac{M_{s} !}{K_{s} !}} \alpha_{s}^{\left(K_{s}-M_{s}\right) / 2} \mathcal{L}_{M_{s}}^{K_{s}-M_{s}}\left(\alpha_{s}\right)
$$

where $\mathcal{L}_{M_{s}}^{K_{s}-M_{s}}\left(\alpha_{s}\right)$ is the associated laguerre polynomial. 


\section{S2 DYADIC GREEN'S FUNCTION OF THREE-LAYER STRUCTURE (DIELECTRIC-COATED SILVER SYSTEM)}

The dyadic Green's function $\overline{\overline{\boldsymbol{G}}}\left(\boldsymbol{r}_{\mathrm{M}}, \boldsymbol{r}_{\mathrm{M}}, \omega\right)$ for a molecule above a dielectric-coated silver system can be decomposed into

$$
\overline{\overline{\boldsymbol{G}}}\left(\boldsymbol{r}_{\mathrm{M}}, \boldsymbol{r}_{\mathrm{M}}, \omega\right)=\overline{\overline{\boldsymbol{G}}}_{0}\left(\boldsymbol{r}_{\mathrm{M}}, \boldsymbol{r}_{\mathrm{M}}, \omega\right)+\overline{\overline{\boldsymbol{G}}}_{\mathrm{s}}\left(\boldsymbol{r}_{\mathrm{M}}, \boldsymbol{r}_{\mathrm{M}}, \omega\right)+\overline{\overline{\boldsymbol{G}}}_{\mathrm{p}}\left(\boldsymbol{r}_{\mathrm{M}}, \boldsymbol{r}_{\mathrm{M}}, \omega\right)
$$

the free-space dyadic Green's function $\overline{\boldsymbol{G}}_{0}\left(\boldsymbol{r}_{\mathrm{A}}, \boldsymbol{r}_{\mathrm{B}}, \omega\right)$ is given by, ${ }^{5,6}$

$$
\overline{\overline{\boldsymbol{G}}}_{0}\left(\boldsymbol{r}_{\mathrm{A}}, \boldsymbol{r}_{\mathrm{B}}, \omega\right)=\frac{e^{i k_{0} R}}{4 \pi k_{0}^{2} R}\left\{\left(\mathbf{I}_{3}-\frac{\boldsymbol{R} \otimes \boldsymbol{R}}{R^{2}}\right) k_{0}^{2}+\left(3 \frac{\boldsymbol{R} \otimes \boldsymbol{R}}{R^{2}}-\mathbf{I}_{3}\right)\left(\frac{1}{R^{2}}-\frac{i k_{0}}{R}\right)\right\},
$$

where $\boldsymbol{R}=\boldsymbol{r}_{\mathrm{A}}-\boldsymbol{r}_{\mathrm{B}}, R=|\boldsymbol{R}|, k_{0}=\omega / c$ is the wavenumber in a vacuum. In this paper, the imaginary part of the free-space dyadic Green's function $\operatorname{Im} \overline{\boldsymbol{G}}_{0}\left(\boldsymbol{r}_{\mathrm{M}}, \boldsymbol{r}_{\mathrm{M}}, \omega\right)$ can be proven, ${ }^{1}$

$$
\operatorname{Im} \overline{\boldsymbol{G}}_{0}\left(\boldsymbol{r}_{\mathrm{M}}, \boldsymbol{r}_{\mathrm{M}}, \omega\right)=\frac{k_{0}}{6 \pi} \mathbf{I}_{3}=\frac{k_{0}}{6 \pi}\left[\begin{array}{ccc}
1 & 0 & 0 \\
0 & 1 & 0 \\
0 & 0 & 1
\end{array}\right]
$$

The s-(p-) polarized dyadic Green's function can be calculated based on

$$
\begin{aligned}
& \overline{\overline{\boldsymbol{G}}}_{\mathrm{s}}\left(\boldsymbol{r}_{\mathrm{M}}, \boldsymbol{r}_{\mathrm{M}}, \omega\right)=\int_{0}^{\infty} \frac{i d q}{4 \pi} R_{\mathrm{s}}(q, \omega) \overline{\overline{\boldsymbol{M}}}_{\mathrm{s}}(q, \omega, 0) e^{2 i K_{z, 0} h_{1}}, \\
& \overline{\overline{\boldsymbol{G}}}_{\mathrm{p}}\left(\boldsymbol{r}_{\mathrm{M}}, \boldsymbol{r}_{\mathrm{M}}, \omega\right)=\int_{0}^{\infty} \frac{i d q}{4 \pi} R_{\mathrm{p}}(q, \omega) \overline{\overline{\boldsymbol{M}}}_{\mathrm{p}}(q, \omega, 0) e^{2 i K_{z, 0} h_{1}},
\end{aligned}
$$

where $K_{z, i}=\sqrt{\epsilon_{\mathrm{r}, i}(\omega) k_{0}^{2}-q^{2}}$ is the z-component wavevector in the media with the relative permittivity (dielectric function) $\epsilon_{\mathrm{r}, i}(\omega)$ (the sub-script $\left.i=0,1,2\right) . R_{\mathrm{s}(\mathrm{p})}(q, \omega)$ is the reflection 
coefficient of $\mathrm{s}-(\mathrm{p}-)$ polarized electric field, and can be expressed as,

$$
\begin{aligned}
& R_{\mathrm{s}(\mathrm{p})}(q, \omega)= \begin{cases}\frac{r_{\mathrm{s}(\mathrm{p}), 01}+r_{\mathrm{s}(\mathrm{p}), 12} e^{2 i K_{z, 1} h_{2}}}{1+r_{\mathrm{s}(\mathrm{p}), 01} r_{\mathrm{s}(\mathrm{p}), 12} e^{2 i K_{z, 1} h_{2}}} & \text { dielectric-coated Ag system } \\
r_{\mathrm{s}(\mathrm{p}), 02} & \text { bulk Ag system }\end{cases} \\
& r_{\mathrm{s}, i j}=\frac{K_{z, i}-K_{z, j}}{K_{z, i}+K_{z, j}} \\
& r_{\mathrm{p}, i j}=\frac{\epsilon_{\mathrm{r}, j}(\omega) K_{z, i}-\epsilon_{\mathrm{r}, i}(\omega) K_{z, j}}{\epsilon_{\mathrm{r}, j}(\omega) K_{z, i}+\epsilon_{\mathrm{r}, i}(\omega) K_{z, j}}
\end{aligned}
$$

The $3 \times 3$ matrices $\overline{\overline{\boldsymbol{M}}}_{\mathrm{s}}(q, \omega, 0)$ and $\overline{\overline{\boldsymbol{M}}}_{\mathrm{p}}(q, \omega, 0)$ in Eq. (S53) are defined

$$
\begin{aligned}
& \overline{\overline{\boldsymbol{M}}}_{\mathrm{s}}(q, \omega, 0)=\frac{q}{2 K_{z, 0}}\left[\begin{array}{lll}
1 & 0 & 0 \\
0 & 1 & 0 \\
0 & 0 & 0
\end{array}\right], \\
& \overline{\overline{\boldsymbol{M}}}_{\mathrm{p}}(q, \omega, 0)=\frac{-q K_{z, 0}}{2 k_{0}^{2}}\left[\begin{array}{ccc}
1 & 0 & 0 \\
0 & 1 & 0 \\
0 & 0 & \frac{-2 q^{2}}{K_{z, 0}^{2}}
\end{array}\right] .
\end{aligned}
$$




\section{S3 DERIVATION OF EQ. (8) UNDER THE HIGH-VIBRATIONAL-FREQUENCY LIMIT}

We define the indexes of high-frequency vibrational modes as $s_{\mathrm{H}}$. If the Huang-Rhys factors for all low-frequency vibrational modes $s_{\mathrm{L}}$ are nearly equal to zero, then in the memory kernel of molecular vibrations $\left\langle K_{s_{\mathrm{L}}}(0) \mid M_{s_{\mathrm{L}}}\left(\alpha_{s_{\mathrm{L}}}\right)\right\rangle=\delta_{K_{s_{\mathrm{L}}}, M_{s_{\mathrm{L}}}}$. Therefore, the memory kernel of molecular vibrations can be simplified as,

$$
\begin{aligned}
& K_{\mathrm{vib}}^{\left\{K_{1}, \cdots, K_{n}\right\} \leftarrow\left\{L_{1}, \cdots, L_{n}\right\}}\left(t, t^{\prime}\right) \\
&=\prod_{s=1}^{n} \sum_{M_{s}=0}^{\infty}\left\langle K_{s}(0) \mid M_{s}\left(\alpha_{s}\right)\right\rangle e^{i \omega_{\mathrm{v}, s}\left(K_{s}-M_{s}\right) t}\left\langle M_{s}\left(\alpha_{s}\right) \mid L_{s}(0)\right\rangle e^{i \omega_{\mathrm{v}, s}\left(M_{s}-L_{s}\right) t^{\prime}} \\
&=\left(\prod_{s_{\mathrm{L}}} \delta_{K_{s_{\mathrm{L}}}, L_{s_{\mathrm{L}}}}\right) \times \\
&\left(\prod_{s_{\mathrm{H}}} \sum_{M_{s_{\mathrm{H}}}=0}^{\infty}\left\langle K_{s_{\mathrm{H}}}(0) \mid M_{s_{\mathrm{H}}}\left(\alpha_{s_{\mathrm{H}}}\right)\right\rangle e^{i \omega_{\mathrm{v}, s_{\mathrm{H}}}\left(K_{s_{\mathrm{H}}}-M_{s_{\mathrm{H}}}\right) t}\left\langle M_{s_{\mathrm{H}}}\left(\alpha_{s_{\mathrm{H}}}\right) \mid L_{s_{\mathrm{H}}}(0)\right\rangle e^{i \omega_{\mathrm{v}, s_{\mathrm{H}}}\left(M_{s_{\mathrm{H}}}-L_{s_{\mathrm{H}}}\right) t^{\prime}}\right) .
\end{aligned}
$$

For convenience, we define the memory kernel of high-frequency molecular vibrations

$K_{\mathrm{vib}}^{\left\{K_{\mathrm{s}_{\mathrm{H}}}\right\} \leftarrow\left\{L_{s_{\mathrm{H}}}\right\}}\left(t, t^{\prime}\right)$ and the memory kernel of low-frequency molecular vibrations $K_{\mathrm{vib}}^{\left\{K_{s_{\mathrm{L}}}\right\} \leftarrow\left\{L_{s_{\mathrm{L}}}\right\}}\left(t, t^{\prime}\right)$ as follows,

$$
\begin{aligned}
& K_{\mathrm{vib}}^{\left\{K_{s_{\mathrm{H}}}\right\} \leftarrow\left\{L_{s_{\mathrm{H}}}\right\}}\left(t, t^{\prime}\right) \\
& =\prod_{s_{\mathrm{H}}} \sum_{M_{s_{\mathrm{H}}}=0}^{\infty}\left\langle K_{s_{\mathrm{H}}}(0) \mid M_{s_{\mathrm{H}}}\left(\alpha_{s_{\mathrm{H}}}\right)\right\rangle e^{i \omega_{\mathrm{v}, s_{\mathrm{H}}}\left(K_{s_{\mathrm{H}}}-M_{s_{\mathrm{H}}}\right) t}\left\langle M_{s_{\mathrm{H}}}\left(\alpha_{s_{\mathrm{H}}}\right) \mid L_{s_{\mathrm{H}}}(0)\right\rangle e^{i \omega_{\mathrm{v}, s_{\mathrm{H}}}\left(M_{s_{\mathrm{H}}}-L_{s_{\mathrm{H}}}\right) t^{\prime}}, \\
& K_{\text {vib }}^{\left\{K_{s_{\mathrm{L}}}\right\} \leftarrow\left\{L_{s_{\mathrm{L}}}\right\}}\left(t, t^{\prime}\right) \\
& =\prod_{s_{\mathrm{L}}} \delta_{K_{s_{\mathrm{L}}}, L_{s_{\mathrm{L}}}} .
\end{aligned}
$$


Plugging Eq. (S58) into the Eq. (1) in the manuscript, we get

$$
\dot{c}_{K_{s_{\mathrm{H}}}}^{\mathrm{ex}}(t)=-\sum_{L_{s_{\mathrm{H}}}=0}^{\infty} \int_{0}^{t} d t^{\prime} K_{\mathrm{pol}}\left(t, t^{\prime}\right) K_{\mathrm{vib}}^{\left\{K_{s_{\mathrm{H}}}\right\} \leftarrow\left\{L_{s_{\mathrm{H}}}\right\}}\left(t, t^{\prime}\right) c_{L_{s_{\mathrm{H}}}}^{\mathrm{ex}}\left(t^{\prime}\right) .
$$

In the derivation of Eq. (S61), the low-frequency mode indexes $\left\{K_{s_{\mathrm{L}}}\right\}$ become dummy indexes and are dropped for clarity. Substituting Eq. (7) in the manuscript into Eq. (S61), we obtain

$$
\begin{aligned}
\dot{c}_{K_{s_{\mathrm{H}}}}^{\mathrm{ex}}(t) & -\sum_{L_{s_{\mathrm{H}}}=0}^{\infty} \int_{0}^{t} d t^{\prime} \sum_{M_{s_{\mathrm{H}}}=0}^{\infty}\left\{\frac{F_{\mathrm{P}}\left(\omega_{\mathrm{SPP}}\right)}{2 \pi} \int_{0}^{\infty} d \omega A_{0}(\omega) \frac{\Gamma^{2}}{\left(\omega-\omega_{\mathrm{SPP}}\right)^{2}+\Gamma^{2}} e^{-i\left(\omega-\omega_{\mathrm{eg}}-\sum_{s_{\mathrm{H}}} \omega_{\mathrm{V}, s_{\mathrm{H}}}\left(K_{s_{\mathrm{H}}}-M_{s_{\mathrm{H}}}\right)\right)\left(t-t^{\prime}\right)}\right\} \\
& \prod_{s_{\mathrm{H}}}\left\{\left\langle K_{s_{\mathrm{H}}}(0) \mid M_{s_{\mathrm{H}}}\left(\alpha_{s_{\mathrm{H}}}\right)\right\rangle\left\langle M_{s_{\mathrm{H}}}\left(\alpha_{s_{\mathrm{H}}}\right) \mid L_{s_{\mathrm{H}}}(0)\right\rangle e^{-i \omega_{\mathrm{v}, s_{\mathrm{H}}}\left(L_{s_{\mathrm{H}}}-K_{s_{\mathrm{H}}}\right) t^{\prime}}\right\} C_{L_{s_{\mathrm{H}}}}^{\mathrm{ex}}\left(t^{\prime}\right) .
\end{aligned}
$$

Eq. (S62) can be simplified when the molecular transition frequency $\omega_{\text {eg }}$ resonates with the SPPs $\omega_{\text {SPP. }}$ First, for the frequency dependent integral $\left(\int_{0}^{\infty} d \omega\right)$ in Eq. (S62), the Lorentz function gives most contributions around $\omega \approx \omega_{\text {SPP }}$, so that the approximation $A_{0}(\omega) \approx$ $A_{0}\left(\omega_{\mathrm{SPP}}\right)$ is valid. Moreover, based on the rotating-wave approximation, $e^{-i\left(\omega-\omega_{\mathrm{eg}}-\sum_{s_{\mathrm{H}}} \omega_{\mathrm{v}, s_{\mathrm{H}}}\left(K_{s_{\mathrm{H}}}-M_{s_{\mathrm{H}}}\right)\right)\left(t-t^{\prime}\right)}$ contributes most to the frequency dependent integral when $\omega-\omega_{\text {eg }}-\sum_{s_{\mathrm{H}}} \omega_{\mathrm{v}, s_{\mathrm{H}}}\left(K_{s_{\mathrm{H}}}-M_{s_{\mathrm{H}}}\right)=$ 0 . The above-mentioned conditions $\omega \approx \omega_{\mathrm{SPP}}, \omega-\omega_{\mathrm{eg}}-\sum_{s_{\mathrm{H}}} \omega_{\mathrm{v}, s_{\mathrm{H}}}\left(K_{s_{\mathrm{H}}}-M_{s_{\mathrm{H}}}\right)=0$, and $\omega_{\text {eg }}=\omega_{\text {SPP }}$ indicate that the frequency dependent integral in Eq. (S62) is nonvanishing only

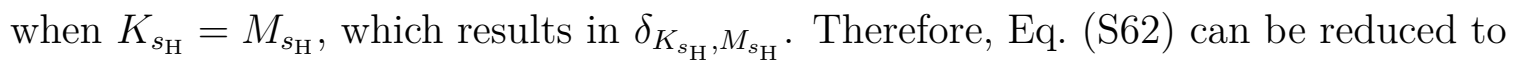

$$
\begin{aligned}
\dot{c}_{K_{s_{\mathrm{H}}}}^{\mathrm{ex}}(t)= & -\sum_{L_{s_{\mathrm{H}}}=0}^{\infty} \int_{0}^{t} d t^{\prime} \sum_{M_{s_{\mathrm{H}}}=0}^{\infty}\left\{\frac{\Gamma}{2} A_{0}\left(\omega_{\mathrm{SPP}}\right) F_{\mathrm{P}}\left(\omega_{\mathrm{SPP}}\right) e^{-\Gamma\left(t-t^{\prime}\right)} \prod_{s_{\mathrm{H}}} \delta_{K_{s_{\mathrm{H}}}, M_{s_{\mathrm{H}}}}\right\} \\
& \prod_{s_{\mathrm{H}}}\left\{\left\langle K_{s_{\mathrm{H}}}(0) \mid M_{s_{\mathrm{H}}}\left(\alpha_{s_{\mathrm{H}}}\right)\right\rangle\left\langle M_{s_{\mathrm{H}}}\left(\alpha_{s_{\mathrm{H}}}\right) \mid L_{s_{\mathrm{H}}}(0)\right\rangle e^{-i \omega_{\mathrm{v}, s_{\mathrm{H}}}\left(L_{s_{\mathrm{H}}}-K_{s_{\mathrm{H}}}\right) t^{\prime}}\right\} C_{L_{s_{\mathrm{H}}}^{\mathrm{ex}}\left(t^{\prime}\right),}
\end{aligned}
$$

where $\delta_{K_{s_{\mathrm{H}}}, M_{s_{\mathrm{H}}}}$ is the Kronecker delta function of vibrational quanta of high-frequency modes. Moreover, in the high-vibrational-frequency limit $\left(\omega_{\mathrm{v}, s_{\mathrm{H}}} \gg \Gamma\right)$, we can apply the rotatingwave approximation for the time dependent integral $\left(\int_{0}^{t} d t^{\prime}\right)$, and Eq. (S63) can be further 
simplified as

$$
\begin{aligned}
\dot{c}_{K_{s_{\mathrm{H}}}}^{\mathrm{ex}}(t)= & -\sum_{L_{s_{\mathrm{H}}}=0}^{\infty} \int_{0}^{t} d t^{\prime} \sum_{M_{s_{\mathrm{H}}}=0}^{\infty}\left\{\frac{\Gamma}{2} A_{0}\left(\omega_{\mathrm{SPP}}\right) F_{\mathrm{P}}\left(\omega_{\mathrm{SPP}}\right) e^{-\Gamma\left(t-t^{\prime}\right)} \prod_{s_{\mathrm{H}}} \delta_{K_{s_{\mathrm{H}}}, M_{s_{\mathrm{H}}}}\right\} \\
& \prod_{s_{\mathrm{H}}}\left\{\left\langle K_{s_{\mathrm{H}}}(0) \mid M_{s_{\mathrm{H}}}\left(\alpha_{s_{\mathrm{H}}}\right)\right\rangle\left\langle M_{s_{\mathrm{H}}}\left(\alpha_{s_{\mathrm{H}}}\right) \mid L_{s_{\mathrm{H}}}(0)\right\rangle \delta_{\left.K_{s_{\mathrm{H}}}, L_{s_{\mathrm{H}}}\right\}}\right\} C_{L_{s_{\mathrm{H}}}}^{\mathrm{ex}}\left(t^{\prime}\right) .
\end{aligned}
$$

It should be emphasized that the rotating-wave approximation eliminates all fast oscillating terms $e^{i \omega_{\mathrm{v}, s_{\mathrm{H}}}\left(K_{s_{\mathrm{H}}}-M_{s_{\mathrm{H}}}\right)\left(t-t^{\prime}\right)}$ with $K_{s_{\mathrm{H}}} \neq M_{s_{\mathrm{H}}}$ and $e^{i \omega_{\mathrm{v}, s_{\mathrm{H}}}\left(K_{s_{\mathrm{H}}}-L_{s_{\mathrm{H}}}\right) t^{\prime}}$ with $L_{s_{\mathrm{H}}} \neq M_{s_{\mathrm{H}}}$ in Eq. (S62). As a result, only the terms which satisfy $L_{s_{\mathrm{H}}}=M_{s_{\mathrm{H}}}=K_{s_{\mathrm{H}}}$ are preserved. When we consider the lowest excited state with zero vibrational quanta $\left(K_{s_{\mathrm{H}}}=0\right.$ for all high-frequency modes), Eq. (S64) becomes

$$
\dot{c}_{0, \cdots, 0}^{\mathrm{ex}}(t)=-\frac{A_{0}\left(\omega_{\mathrm{SPP}}\right) F_{\mathrm{P}}\left(\omega_{\mathrm{SPP}}\right) \Gamma}{2} \prod_{s_{\mathrm{H}}}\left\langle 0(0) \mid 0\left(\alpha_{s_{\mathrm{H}}}\right)\right\rangle\left\langle 0\left(\alpha_{s_{\mathrm{H}}}\right) \mid 0(0)\right\rangle \int_{0}^{t} d t^{\prime} e^{-\Gamma\left(t-t^{\prime}\right)} C_{0, \cdots, 0}^{\mathrm{ex}}\left(t^{\prime}\right) .
$$

Differentiation of Eq. (S65) with respect to $t$, one can obtain the second-order differential equation and Eq. (8) in the manuscript,

$$
\frac{d^{2} c_{0, \cdots, 0}^{\mathrm{ex}}(t)}{d t^{2}}+\Gamma \frac{d c_{0, \cdots, 0}^{\mathrm{ex}}(t)}{d t}+\left(\frac{\Omega}{2}\right)^{2} c_{0, \cdots, 0}^{\mathrm{ex}}(t)=0
$$

where

$$
\Omega=\left(\prod_{s_{\mathrm{H}}}\left|\left\langle 0(0) \mid 0\left(\alpha_{s_{\mathrm{H}}}\right)\right\rangle\right|\right) \sqrt{2 A_{0}\left(\omega_{\mathrm{SPP}}\right) F_{\mathrm{P}}\left(\omega_{\mathrm{SPP}}\right) \Gamma} .
$$




\section{S4 ORIGIN OF LARGER $D_{\mathrm{T}}$ IN NACL-COATED SILVER SYSTEM}

The Purcell factor $F_{\mathrm{P}}(\omega)$ is calculated at different distances $d=h_{1}+h_{2}$ for bulk Ag and $\mathrm{NaCl}$-coated Ag systems based on Eqs. (4) and (5) in the manuscript. We subsequently use the Lorentz function Eq. (8) to fit the calculated $F_{\mathrm{P}}(\omega)$. The distance dependence of the fitting parameters $F_{\mathrm{P}}\left(\omega_{\mathrm{SPP}}\right), \omega_{\mathrm{SPP}}$, and $\Gamma$ is plotted as follows,
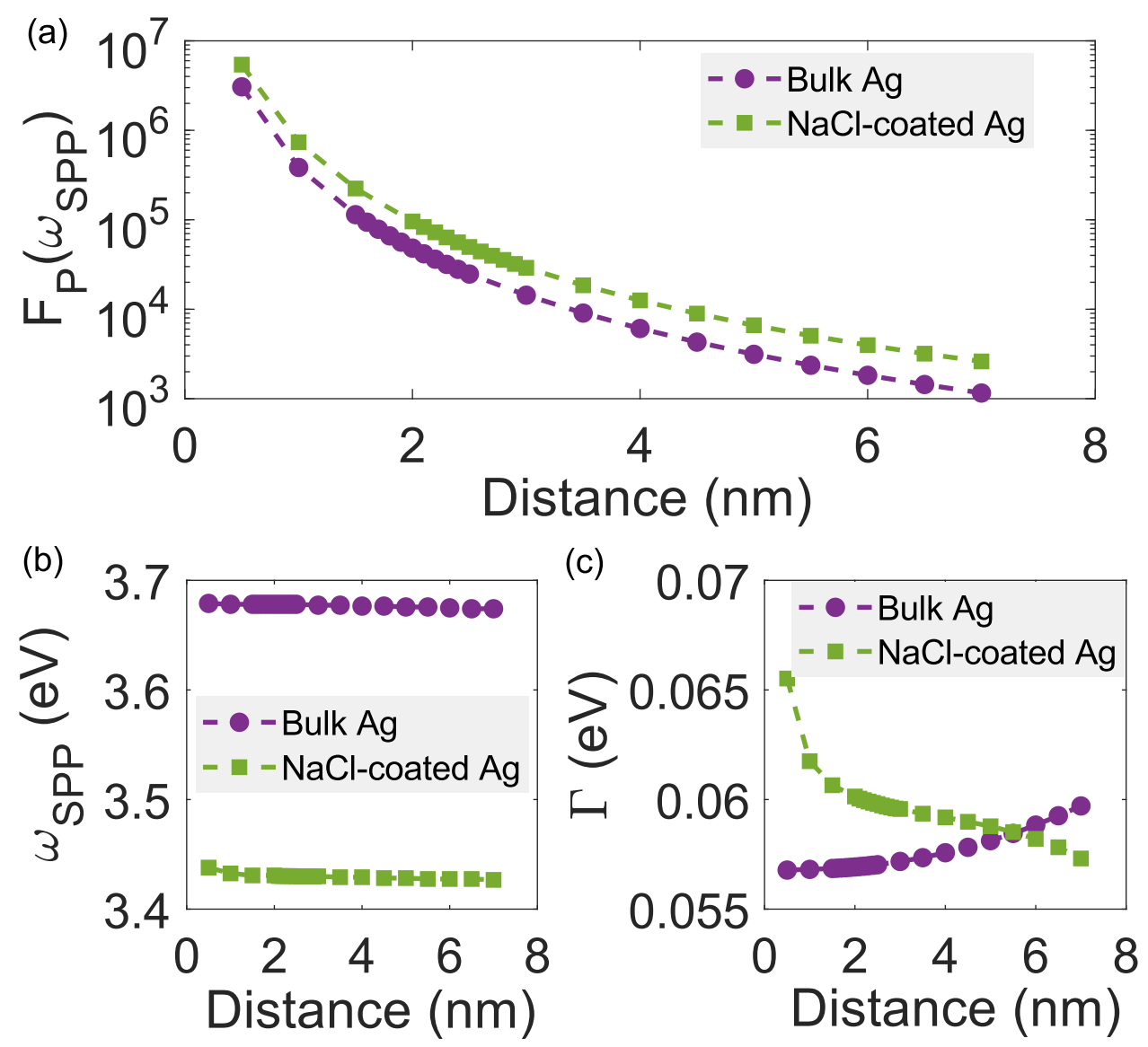

Figure S1: Parameters of the Lorentz function at different distances $d=h_{1}+h_{2}$ above the silver surface. (a) The peak height $F_{\mathrm{P}}\left(\omega_{\mathrm{SPP}}\right)$ with the distance. (b) The peak frequency $\omega_{\text {SPP }}$ with the distance. (c) The half width at half maximum $\Gamma$ versus the distance.

Fig. (S1)c clearly shows that $\Gamma$ is less sensitive to distance $d$ and the property of the thin film $\epsilon_{\mathrm{r}, 1}(\omega)$. On the other hand, it is evident that peak height $F_{\mathrm{P}}\left(\omega_{\mathrm{SPP}}\right)$ of the NaCl-coated $\mathrm{Ag}$ system is always above the bulk Ag system. Because of the criteria of the coherent dynamics $\Omega>\Gamma$ and $\Omega \propto \sqrt{F\left(\omega_{\mathrm{SPP}}\right)}$, the quantum dynamics of molecular fluorescence 
in the $\mathrm{NaCl}$-coated silver system should be always more coherent than that in the bulk silver system, which leads to a larger coherent-to-incoherent transition distance $d_{\mathrm{T}}$ for the $\mathrm{NaCl}$-coated silver system. 


\section{S5 PURCELL FACTOR FOR DIELECTRIC-COATED SYSTEMS WITH EXPERIMENTAL DIELECTRIC FUNCTIONS AND APPROXIMATED DIELECTRIC CONSTANTS}

Purcell factors for different dielectric-coated systems are calculated with experimental dielectric functions $\epsilon_{\mathrm{r}, 1}(\omega)$ and approximated dielectric constants $\epsilon_{\mathrm{r}, 1}$. The experimental dielectric function of $\mathrm{NaCl}$ is adopted from the work of $\mathrm{Li}^{7}{ }^{7}$ the experimental dielectric function of $\mathrm{SiO}_{2}$ is adopted from the work of Ghosh ${ }^{8}$ the experimental dielectric function of $\mathrm{MgF}_{2}$ is adopted from the work of Dodge; ${ }^{9}$ the experimental dielectric function of $\mathrm{Al}_{2} \mathrm{O}_{3}$ is adopted from the work of Malitson et al. ${ }^{10}$
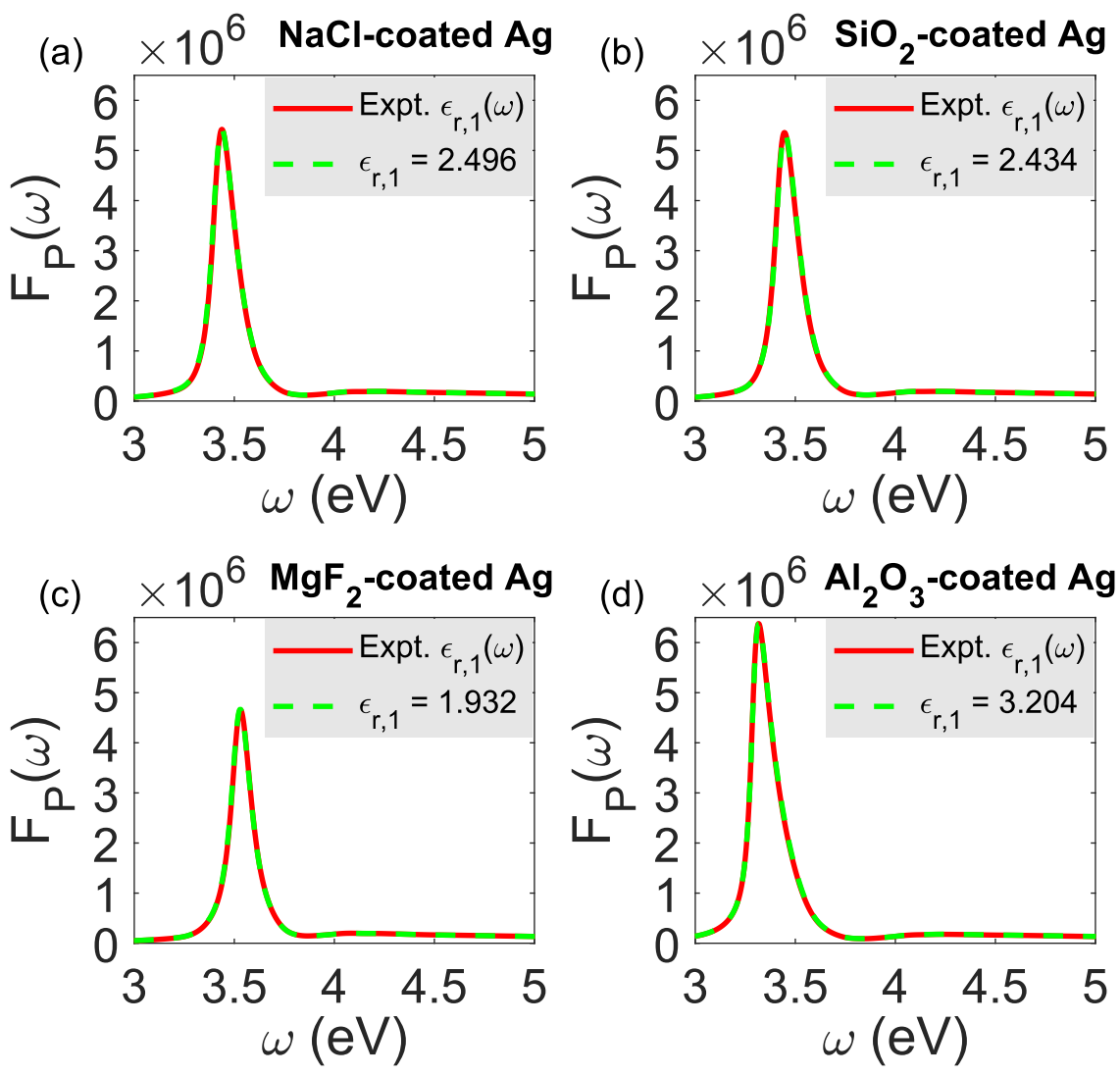

Figure S2: Purcell factors for different dielectric-coated systems. (a) NaCl-coated silver system. (b) $\mathrm{SiO}_{2}$-coated silver system. (c) $\mathrm{MgF}_{2}$-coated silver system. (d) $\mathrm{Al}_{2} \mathrm{O}_{3}$-coated silver system. 
The coincidences of the red solid lines (based on the experimental dielectric functions $\epsilon_{\mathrm{r}, 1}(\omega)$ ) and the green dash lines (based on the approximated dielectric constants $\epsilon_{\mathrm{r}, 1}$ ) in Figures (S2)a-d indicate that one could use dielectric constants to approximate dielectric functions for transparent materials. 


\section{S6 DIELECTRIC FUNCTION OF SILVER BASED ON DRUDE-SOMMERFELD MODEL}

An external electric field $\boldsymbol{E}(t)=\boldsymbol{E}_{0} e^{-i \omega t}$ leads to a displacement $\boldsymbol{r}_{\mathrm{e}}$ of the free electron of silver. Based on the Drude-Sommerfeld model, $\boldsymbol{r}_{\mathrm{e}}$ follows the dynamics

$$
m_{\mathrm{e}} \frac{d^{2} \boldsymbol{r}_{\mathrm{e}}}{d t^{2}}+m_{\mathrm{e}} \Gamma_{\mathrm{e}} \frac{d \boldsymbol{r}_{\mathrm{e}}}{d t}=e \boldsymbol{E}_{0} e^{-i \omega t},
$$

where $m_{\mathrm{e}}$ and $e$ are the effective mass and the charge of the free electrons, respectively. $\Gamma_{\mathrm{e}}$ is the damping term describing the scattering process between the free electron and nucleus. ${ }^{11}$ Eq. (S68) can be solved with an ansatz $\boldsymbol{r}_{\mathrm{e}}(t)=\boldsymbol{r}_{0} e^{-i \omega t}$,

$$
\boldsymbol{r}_{\mathrm{e}}(t)=\frac{-e \boldsymbol{E}_{0} / m_{\mathrm{e}}}{\omega^{2}+i \Gamma_{\mathrm{e}} \omega} e^{-i \omega t} .
$$

The macroscopic polarization $\boldsymbol{P}(\omega)$ of silver is related to the displacement of the free electron in the frequency domain $\boldsymbol{r}_{\mathrm{e}}(\omega)$,

$$
\boldsymbol{P}(\omega)=n e \boldsymbol{r}_{\mathrm{e}}(\omega)=\frac{-n e^{2} \boldsymbol{E}_{0} / m_{\mathrm{e}}}{\omega^{2}+i \Gamma_{\mathrm{e}} \omega} \delta(\omega),
$$

where $n$ is the free electron density of silver, and the dielectric function (relative permittivity) $\epsilon_{\mathrm{Ag}}(\omega)$ can be derived by using the constitutive relations

$$
\boldsymbol{D}(\omega)=\epsilon_{0} \epsilon_{\mathrm{Ag}}(\omega) \boldsymbol{E}(\omega)=\epsilon_{0} \boldsymbol{E}(\omega)+\boldsymbol{P}(\omega),
$$

where $\boldsymbol{D}(\omega)$ is the electric displacement field in the frequency domain, and $\boldsymbol{E}(\omega)=\boldsymbol{E}_{0} \delta(\omega)$ is the electric field in the frequency domain. Therefore, we can derive the dielectric function 
$\epsilon_{\mathrm{Ag}}^{\mathrm{DS}}(\omega)$ based on the Drude-Sommerfeld model,

$$
\epsilon_{\mathrm{Ag}}^{\mathrm{DS}}(\omega)=1-\frac{\omega_{\mathrm{p}}^{2}}{\omega^{2}+i \Gamma_{\mathrm{e}} \omega},
$$

where $\omega_{\mathrm{p}}=\sqrt{n e^{2} /\left(\epsilon_{0} m_{\mathrm{e}}\right)}$ is the volume plasma frequency. In order to fit the dielectric function with experimental data in the optical range, one has to introduce a constant offset $\epsilon_{\infty}$ to Eq. (S72) due to the contributions of interband transitions which are not considered in the original Drude-Sommerfeld theory. ${ }^{12}$ The modified dielectric function based on the Drude-Sommerfeld model is

$$
\epsilon_{\mathrm{Ag}}(\omega)=\epsilon_{\infty}-\frac{\omega_{\mathrm{p}}^{2}}{\omega^{2}+i \Gamma_{\mathrm{e}} \omega}
$$

In this paper, we set $\epsilon_{\infty}=5.3, \omega_{\mathrm{p}}=9.5 \mathrm{eV}$, and $\Gamma_{\mathrm{e}}=0.2 \mathrm{eV}$ to fit the experimental dielectric function of silver. ${ }^{13}$ 


\section{S7 DERIVATION OF EQ. (11) WITH QUASI-STATIC APPROXIMATION AND DIELECTRIC FUNCTION OF SILVER BASED ON DRUDE-SOMMERFELD MODEL}

The transition dipole moment of the molecule is oriented in the $z$ direction $\boldsymbol{\mu}=\left(0,0, \mu_{z}\right)$ in this paper, so the spontaneous emission rate in media $A_{\mathrm{FG}}(\omega)$ becomes

$$
A_{\mathrm{FG}}(\omega)=\frac{2 \omega^{2}}{\hbar c^{2} \epsilon_{0}} \mu_{z}^{2} \operatorname{Im} G_{z z}\left(\boldsymbol{r}_{\mathrm{M}}, \boldsymbol{r}_{\mathrm{M}}, \omega\right)
$$

where $\operatorname{Im} G_{z z}\left(\boldsymbol{r}_{\mathrm{M}}, \boldsymbol{r}_{\mathrm{M}}, \omega\right)$ is the $z z$ component of the imaginary part of the dyadic Green's function $\overline{\overline{\boldsymbol{G}}}\left(\boldsymbol{r}_{\mathrm{M}}, \boldsymbol{r}_{\mathrm{M}}, \omega\right)$. Based on Eq. (S50), Eq. (S52), Eq. (S53), and Eq. (S57), we get

$$
\begin{aligned}
A_{\mathrm{FG}}(\omega) & =\frac{2 \omega^{2} \mu_{z}^{2}}{\hbar c^{2} \epsilon_{0}} \operatorname{Im}\left\{G_{0, z z}\left(\boldsymbol{r}_{\mathrm{M}}, \boldsymbol{r}_{\mathrm{M}}, \omega\right)+G_{\mathrm{p}, z z}\left(\boldsymbol{r}_{\mathrm{M}}, \boldsymbol{r}_{\mathrm{M}}, \omega\right)\right\} \\
& =A_{0}(\omega)+\frac{2 \omega^{2} \mu_{z}^{2}}{\hbar \epsilon_{0} c^{2}} \operatorname{Im}\left\{G_{\mathrm{p}, z z}\left(\boldsymbol{r}_{\mathrm{M}}, \boldsymbol{r}_{\mathrm{M}}, \omega\right)\right\},
\end{aligned}
$$

where $\operatorname{Im} G_{0, z z}\left(\boldsymbol{r}_{\mathrm{M}}, \boldsymbol{r}_{\mathrm{M}}, \omega\right)=k_{0} /(6 \pi)$ is used, as shown in Eq. (S52). $k_{0}=\omega / c$ is the wavenumber in a vacuum. $A_{0}(\omega)$ is defined in Eq. (5) in the manuscript. $G_{\mathrm{p}, z z}\left(\boldsymbol{r}_{\mathrm{M}}, \boldsymbol{r}_{\mathrm{M}}, \omega\right)$ can be derived by using Eqs. (S53) and (S57), and the result is

$$
G_{\mathrm{p}, z z}\left(\boldsymbol{r}_{\mathrm{M}}, \boldsymbol{r}_{\mathrm{M}}, \omega\right)=\frac{i}{4 \pi k_{0}^{2}} \int_{0}^{\infty} d q \frac{q^{3} R_{\mathrm{p}}(q, \omega)}{K_{z, 0}} e^{2 i K_{z, 0} h_{1}}
$$

In principle, Eq. (S76) can only be solved numerically, but when the molecule is placed very near the surface, we can apply the quasi-static approximation to evaluate it analytically. By setting $q \rightarrow+\infty$, the Fresnel coefficients $r_{\mathrm{p}, i j}$ in Eq. (S56) is approximated as

$$
\begin{aligned}
r_{\mathrm{p}, i j} & =\frac{\epsilon_{\mathrm{r}, j} \sqrt{\epsilon_{\mathrm{r}, i}(\omega) k_{0}^{2}-q^{2}}-\epsilon_{\mathrm{r}, i}(\omega) \sqrt{\epsilon_{\mathrm{r}, j} k_{0}^{2}-q^{2}}}{\epsilon_{\mathrm{r}, j}(\omega) \sqrt{\epsilon_{\mathrm{r}, i} k_{0}^{2}-q^{2}}+\epsilon_{\mathrm{r}, i}(\omega) \sqrt{\epsilon_{\mathrm{r}, j} k_{0}^{2}-q^{2}}} \approx \frac{i q \epsilon_{\mathrm{r}, j}(\omega)-i q \epsilon_{\mathrm{r}, i}(\omega)}{i q \epsilon_{\mathrm{r}, j}(\omega)+i q \epsilon_{\mathrm{r}, i}(\omega)} \\
& =\frac{\epsilon_{\mathrm{r}, j}(\omega)-\epsilon_{\mathrm{r}, i}(\omega)}{\epsilon_{\mathrm{r}, j}(\omega)+\epsilon_{\mathrm{r}, i}(\omega)}
\end{aligned}
$$

The reflection coefficient $R_{\mathrm{p}}(q, \omega)$ in Eq. (S54) can be expanded in terms of a geometric 
series, ${ }^{14,15}$

$$
\begin{aligned}
R_{\mathrm{p}}(q, \omega) & =\frac{r_{\mathrm{p}, 01}+r_{\mathrm{p}, 12} e^{2 i K_{z, 1} h_{2}}}{1-r_{\mathrm{p}, 10} r_{\mathrm{p}, 12} e^{2 i K_{z, 1} h_{2}}} \\
& =\left(r_{\mathrm{p}, 01}+r_{\mathrm{p}, 12} e^{2 i K_{z, 1} h_{2}}\right) \sum_{m=0}^{\infty}\left(r_{\mathrm{p}, 10} r_{\mathrm{p}, 12} e^{2 i K_{z, 1} h_{2}}\right)^{m} \\
& \approx\left(r_{\mathrm{p}, 01}+r_{\mathrm{p}, 12} e^{2 i K_{z, 1} h_{2}}\right)\left(1+r_{\mathrm{p}, 10} r_{\mathrm{p}, 12} e^{2 i K_{z, 1} h_{2}}\right) \\
& \approx r_{\mathrm{p}, 01}+\left(1-r_{\mathrm{p}, 01}^{2}\right) r_{\mathrm{p}, 12} e^{2 i K_{z, 1} h_{2}},
\end{aligned}
$$

the first term gives a relatively small contribution, so we only keep the second term,

$$
R_{\mathrm{p}}(q, \omega) \approx\left(1-r_{\mathrm{p}, 01}^{2}\right) r_{\mathrm{p}, 12} e^{-2 q h_{2}}
$$

Substituting Eq. (S79) into Eq. (S76), we can evaluate the integral analytically,

$$
\begin{aligned}
G_{\mathrm{p}, z z}\left(\boldsymbol{r}_{\mathrm{M}}, \boldsymbol{r}_{\mathrm{M}}, \omega\right) & \approx \frac{i}{4 \pi k_{0}^{2}}\left(1-r_{\mathrm{p}, 01}^{2}\right) r_{\mathrm{p}, 12} \int_{0}^{\infty} d q \frac{q^{3}}{i q} e^{-2 q\left(h_{1}+h_{2}\right)} \\
& =\frac{1}{4 \pi k_{0}^{2}}\left(1-r_{\mathrm{p}, 01}^{2}\right) r_{\mathrm{p}, 12} \frac{1}{4\left(h_{1}+h_{2}\right)^{3}}
\end{aligned}
$$

where $r_{\mathrm{p}, 01}=\left(\epsilon_{\mathrm{r}, 1}-1\right) /\left(\epsilon_{\mathrm{r}, 1}+1\right), r_{\mathrm{p}, 12}=\left(\epsilon_{\mathrm{Ag}}(\omega)-\epsilon_{\mathrm{r}, 1}\right) /\left(\epsilon_{\mathrm{Ag}}(\omega)+\epsilon_{\mathrm{r}, 1}\right)$ (the top layer is vacuum and the bottom layer is silver, so that $\epsilon_{\mathrm{r}, 0}=1$ and $\left.\epsilon_{\mathrm{r}, 2}(\omega)=\epsilon_{\mathrm{Ag}}(\omega)\right)$, and the Purcell factor can be analytically expressed as

$$
\begin{aligned}
F_{\mathrm{P}}(\omega) & =\frac{A_{\mathrm{FG}}(\omega)}{A_{0}(\omega)} \\
& =1+\frac{6 \pi \mu_{z}^{2}}{k_{0}|\boldsymbol{\mu}|^{2}} \operatorname{Im}\left\{G_{\mathrm{p}, z z}\left(\boldsymbol{r}_{\mathrm{M}}, \boldsymbol{r}_{\mathrm{M}}, \omega\right)\right\} \\
& =1+\frac{6 \pi \mu_{z}^{2}}{k_{0}|\boldsymbol{\mu}|^{2}} \operatorname{Im}\left\{\frac{1}{4 \pi k_{0}^{2}} \frac{\left(1-r_{\mathrm{p}, 01}^{2}\right) r_{\mathrm{p}, 12}}{4\left(h_{1}+h_{2}\right)^{3}}\right\} \\
& \approx\left[1-\left(\frac{\epsilon_{\mathrm{r}, 1}-1}{\epsilon_{\mathrm{r}, 1}+1}\right)^{2}\right] \frac{3}{4 k_{0}^{3}\left(h_{1}+h_{2}\right)^{3}} \frac{\epsilon_{\mathrm{r}, 1} \operatorname{Im}\left\{\epsilon_{\mathrm{Ag}}(\omega)\right\}}{\left|\epsilon_{\mathrm{Ag}}(\omega)+\epsilon_{\mathrm{r}, 1}\right|^{2}}
\end{aligned}
$$

If we apply the dielectric function of silver based on the Drude-Sommerfeld model, i.e., 
Eq. (S73), the Purcell factor can be further simplified as,

$$
\begin{aligned}
F_{\mathrm{P}}(\omega) & \approx\left[1-\left(\frac{\epsilon_{\mathrm{r}, 1}-1}{\epsilon_{\mathrm{r}, 1}+1}\right)^{2}\right] \frac{3}{4 k_{0}^{3}\left(h_{1}+h_{2}\right)^{3}} \frac{\epsilon_{\mathrm{r}, 1} \frac{\Gamma_{\mathrm{e}} \omega_{\mathrm{p}}^{2}}{\omega\left(\omega^{2}+\Gamma_{\mathrm{e}}^{2}\right)}}{\frac{\omega^{2}\left[\left(\epsilon_{\infty}+\epsilon_{\mathrm{r}, 1}\right)\left(\omega^{2}+\Gamma_{\mathrm{e}}^{2}\right)-\omega_{\mathrm{p}}^{2}\right]^{2}+\Gamma_{\mathrm{e}}^{2} \omega_{\mathrm{P}}^{4}}{\omega^{2}\left(\omega^{2}+\Gamma_{\mathrm{e}}^{2}\right)^{2}}} \\
& =\left[1-\left(\frac{\epsilon_{\mathrm{r}, 1}-1}{\epsilon_{\mathrm{r}, 1}+1}\right)^{2}\right] \frac{3}{4 k_{0}^{3}\left(h_{1}+h_{2}\right)^{3}} \frac{\epsilon_{\mathrm{r}, 1} \omega_{\mathrm{c}}^{3}}{\omega_{\mathrm{p}}^{2}} \frac{\omega_{\mathrm{c}} \Gamma_{\mathrm{e}}\left(\omega^{2}+\Gamma_{\mathrm{e}}^{2}\right) \omega}{\omega^{2}\left[\omega^{2}+\Gamma_{\mathrm{e}}^{2}-\omega_{\mathrm{c}}^{2}\right]^{2}+\Gamma_{\mathrm{e}}^{2} \omega_{\mathrm{c}}^{4}},
\end{aligned}
$$

where $\omega_{\mathrm{c}}=\omega_{\mathrm{p}} / \sqrt{\epsilon_{\mathrm{r}, 1}+\epsilon_{\infty}}$, and we have numerically proven that Eq. (S82) behaves like a Lorentzian (when $\epsilon_{\infty}=5.3, \omega_{\mathrm{p}}=9.5 \mathrm{eV}$, and $\Gamma_{\mathrm{e}}=0.2 \mathrm{eV}$ )

$$
F_{\mathrm{P}}(\omega) \approx\left[1-\left(\frac{\epsilon_{\mathrm{r}, 1}-1}{\epsilon_{\mathrm{r}, 1}+1}\right)^{2}\right] \frac{3 c^{3}}{4 \omega^{3}\left(h_{1}+h_{2}\right)^{3}} \frac{\epsilon_{\mathrm{r}, 1} \omega_{\mathrm{c}}^{3}}{\Gamma_{\mathrm{e}} \omega_{\mathrm{p}}^{2}} \frac{\left(\Gamma_{\mathrm{e}} / 2\right)^{2}}{\left(\omega-\omega_{\mathrm{c}}\right)^{2}+\left(\Gamma_{\mathrm{e}} / 2\right)^{2}}
$$




\section{References}

(1) Dung, H. T.; Knöll, L.; Welsch, D.-G. Spontaneous decay in the presence of dispersing and absorbing bodies: General theory and application to a spherical cavity. Phys. Rev. A 2000, 62, 053804.

(2) Dung, H. T.; Knöll, L.; Welsch, D.-G. Three-dimensional quantization of the electromagnetic field in dispersive and absorbing inhomogeneous dielectrics. Phys. Rev. A 1998, 57, 3931-3942.

(3) Fischer, G. Vibronic Coupling: The Interaction Between the Electronic and Nuclear Motions; Theoretical chemistry; Academic Press, 1984.

(4) Fleming, C.; Cummings, N. I.; Anastopoulos, C.; Hu, B. L. The rotating-wave approximation: consistency and applicability from an open quantum system analysis. J. Phys. A Math. Theor. 2010, 43, 405304.

(5) Jackson, J. D. Classical electrodynamics; American Association of Physics Teachers, 1999.

(6) Wu, J.-S.; Lin, Y.-C.; Sheu, Y.-L.; Hsu, L.-Y. Characteristic Distance of Resonance Energy Transfer Coupled with Surface Plasmon Polaritons. J. Phys. Chem. Lett. 2018, 9, 7032-7039.

(7) Li, H. H. Refractive index of alkali halides and its wavelength and temperature derivatives. J. Phys. Chem. Ref. Data 1976, 5, 329-528.

(8) Ghosh, G. Dispersion-equation coefficients for the refractive index and birefringence of calcite and quartz crystals. Opt. Commun. 1999, 163, 95-102.

(9) Dodge, M. J. Refractive properties of magnesium fluoride. Appl. Opt. 1984, 23, 1980.

(10) Malitson, I. H.; Dodge, M. J. Refractive-index and birefringence of synthetic sapphire. J. Opt. Soc. Am. 1972; p 1405. 
(11) Novotny, L.; Hecht, B. Principles of Nano-Optics; Cambridge University Press: Cambridge, 2012.

(12) González-Tudela, A.; Huidobro, P. A.; Martín-Moreno, L.; Tejedor, C.; GarcíaVidal, F. J. Reversible dynamics of single quantum emitters near metal-dielectric interfaces. Phys. Rev. B 2014, 89, 041402.

(13) Johnson, P. B.; Christy, R. W. Optical Constants of the Noble Metals. Phys. Rev. B 1972, 6, 4370-4379.

(14) Chew, W. C.; Kong, J. A. Electromagnetic field of a dipole on a two-layer earth. GEOPHYSICS 1981, 46, 309-315.

(15) Chew, W. C. Waves and fields in inhomogeneous media; IEEE press, 1995. 